\title{
Non equilibrium dynamics of isolated disordered systems: the classical Hamiltonian p-spin model
}

\author{
Leticia F Cugliandolo ${ }^{1}$, Gustavo S Lozano \\ and Emilio N Nessi ${ }^{2,3}$
}

Sorbonne Universités, Université Pierre et Marie Curie-Paris 6. Laboratoire de Physique Théorique et Hantes Energies, 4, Place Jussieu, 75252 Paris Cedex 05, France

2 Departamento de Física, FCEYN Universidad de Buenos Aires \& IFIBA CONICET, Pabellón 1 Ciudad Universitaria, 1428 Buenos Aires, Argentina

E-mail: leticia@ilpthe.jussieu.fr, lozano@df.uba.ar and unessiôdf.uba.ar

Received 30 March 2017

Accepted for publication 23 June 2017

Published 23 August 2017

Online at stacks.iop.org/JSTAT/2017/083301

https://doi.org/10.1088/1742-5468/a.a.7dfb

\begin{abstract}
We study the dynamics of a classical disordered macroscopic model completely isolated from the environment reproducing, in a classical setting, the 'quantum quench' protocol. We show that, depending on the pre and post quench parameters, the system approaches equilibrium, succeeding to act as a bath on itself, or remains out of equilibrium, in two different ways. In the latter one, the system stays confined in a metastable state in which it undergoes stationary dynamics characterised by a single temperature. In the other, the system ages and its dynamics are characterised by two temperatures associated with observations made at short and long time differences (high and low frequencies). The parameter dependence of the asymptotic states is rationalised in terms of a dynamic phase diagram with one equilibrium and two out of equilibrium phases. Aspects of pre-thermalisation are observed and discussed. Similarities and differences with the dynamics of the dissipative model are also cxplained.
\end{abstract}

Keywords: spin glasses, ergodicity breaking, quantum quenches

\footnotetext{
${ }^{3}$ Author to whom any correspondence should be addressed.
} 


\section{Contents}

1. Introduction 3

2. Background 4

2.1. The Hamiltonian spherical $p$-spin model . . . . . . . . . . . . 4

2.2. The $p \geqslant 3$ case . . . . . . . . . . . . . . . . . 6

2.2.1. Equilibrium. . . . . . . . . . . . . . . . 7

2.2.2. Metastability. . . . . . . . . . . . . . . . 8

2.2.3. Relaxation dynamics . . . . . . . . . . . . . . . . . . . . . . .

3. Quenches and dynamics of the isolated system 13

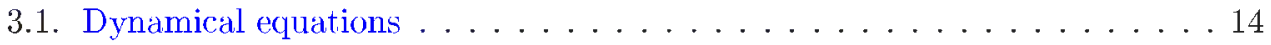

3.2. The Lagrange multiplier . . . . . . . . . . . . . . . . . 17

3.3. Energy change . . . . . . . . . . . . . . . . 18

3.3.1. The energy change after a potential energy quench. . . . . . . . . . 18

3.3.2. The energy change after a quench in the mass . . . . . . . . . 19

4. Asymptotic analysis 20

4.1. Stationary dynamics . . . . . . . . . . . . . . . 21

4.1.1. The asymptotic Lagrange multiplier and the total energy. . . . . . 22

4.1.2. The asymptotic analysis of the correlation equation . . . . . . . 23

4.1.3. The equations fixing the parameters $q, q_{0}$ and $T_{f} \ldots \ldots \ldots 24$

4.1.4. Quench dynamics, target paramagnetic state . . . . . . . . . . . 25

4.1.5. Dynamics within metastable states . . . . . . . . . . . . . . . . . . 25

4.2. Non stationary dynamics and ageing. . . . . . . . . . . . . . 28

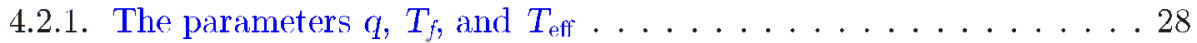

4.3. Summary of asymptotic solutions . . . . . . . . . . . . 29

5. Numerical results 30

5.1. Equilibrium dynamics. . . . . . . . . . . . . . . . . . 30

5.2. Energy injection . . . . . . . . . . . . . . . . . . 31

5.2.1. $T_{s}^{0}<T^{\prime}<T_{d}^{0}$ : from TAP to PM . . . . . . . . . . . 31

5.2.2. $T_{s}^{0}<T^{\prime}<T_{d}^{0}$ : from TAP to TAP . . . . . . . . . . 32

5.2.3. $T_{s}^{0}<T^{\prime}<T_{d}^{0}$ : from TAP to spinodal, transient dynamics. . . . . 33

5.2.4. $T_{s}^{0}<T^{\prime}<T_{d}^{0}$ : from TAP to threshold? . . . . . . . . . 36

5.3. Energy extraction . . . . . . . . . . . . . . . . 37

5.3.1. $T_{s}^{0}<T^{\prime}<T_{d}^{0}$ : from TAP to TAP . . . . . . . . . . . 37

5.3.2. $T^{\prime}>T_{d}^{0}$ : from PM to threshold, ageing dynamics. . . . . . . 37

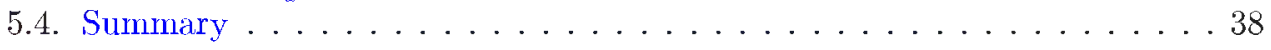

6. The phase diagram $\quad 39$

6.1. The PM-ageing boundary line. . . . . . . . . . . . . . . . . . . . . . . . .

6.2. The TAP-PM boundary line. . . . . . . . . . . . . . . . . . 42

https://doi.org/10.1088/1742-5468/aa7dfb 2 
Non equilibrium dynamics of isolated disordered systems: the classical Hamiltonian p-spin model

7. Conclusions

Acknowledgments

References

\section{Introduction}

The dynamics of isolated many-body quantum systems, and especially the search of a statistical description of their asymptotic evolution [1], are currently receiving great attention. One of the motivations to study these issues theoretically is the practical realisation of quenches of isolated ultra-cold atoms trapped in optical lattices [2]. Another reason for the interest in these questions, is the recently proposed many-body localisation phenomenon in systems with quenched disorder [3].

A quantum quench is the protocol whereby the ground state (or, more generally, a mixed state) of a Hamiltonian $H_{0}$ is unitarily evolved with a different Hamiltonian $H$. A similar procedure can also be realised in a classical setting. It amounts to evolving an isolated system initially prepared in the equilibrium state (or, more generally, a metastable state) of a Hamiltonian $H_{0}$ with a Hamiltonian $H$ with the same form but different parameters. The latter problem has not received as much attention as the former, although we will show that it raises very similar questions to its quantum partner.

The most natural question to pose in the context of quenches of isolated classical or quantum many-body physics is whether the system is able to provide a bath for itsclf, allowing it to reach cquilibration thanks to the interactions. In other words, the question is whether the system attains, in the long time limit, a stationary state of the Gibbs-Boltzmann thermal kind.

Two classes of quantum systems in which the answer to this question is negative have already been identified. These are integrable systems, with a macroscopic number of conserved quasi-local quantities [4], and many-body localised quantum systems, which remain localised in states that are close to their initial conditions forced by the frozen randomness $[5,6]$. The non-ergodic behaviour of these systems is expected to be destabilised by the coupling to an external environment acting as a thermal bath [7].

Our aim is to prove that another class of isolated non-ergodic models exists that are not able to act as baths for themselves. These are frustrated models with complex free-energy landscapes that include, for wide ranges of variation of their parameters, fully trapping regions. For the sake of clarity, and with the aim of distinguishing effects that may be of unique quantum origin from features that are just due to the isolation of the model and/or the peculiar character of the interactions, we start by treating a classical model.

In this paper we start a series of studies of the quench dynamics of isolated classical and quantum interacting disordered models of mean-field kind, that is to say, models with $N$ fully-connected variables, endowed with a quenched random potential and kinetic energy. Their choice is motivated by the fact that their equilibrium, metastable and dissipative dynamics are very well understood and realise the complex free-energy landscape able to render the dynamics non-ergodic. Concretely, they are models with 
Non equilibrium dynamics of isolated disordered systems: the classical Hamiltonian p-spin model

random interactions between $p$ spins that have been extensively used as a mean-field description of the glassy arrest and glassy phenomenology [8-10]. In the infinite $p$ limit they become the random energy model $[11,12\rceil$, also used in the context of many-body localisation studies $[13,14]$. Moreover, there may be deep connections between the glassy physics studied using these kind of models and the physics of disordered electrons undergoing a metal-insulator transition [15].

We show that, under different quenching conditions, the isolated dynamics of these non-integrable interacting systems asymptotically approach:

(i) A paramagnetic stationary state described by a single temperature $T_{f}$, itself determined by the final energy of the system $e_{f}$.

(ii) A metastable state with stationary dynamies. Whether this steady state can or cannot be considered one of Gibbs-Boltzmann equilibrium, is a subtle issue that we will explain in the paper.

(iii) A non-stationary ageing state described by two temperatures, $T_{f}$ and $T_{\text {eff, }}$, that are also related to the final energy of the system $e_{f}$ and other non-trivial properties of it. In this case, the system is clearly out of equilibrium.

The impossibility to relax to thermal equilibrium is related to two prominent features of the potential energy landscape. In case (ii), the system reaches stationarity within one (out of many) metastable state, which can be visualised as disconnected lakes in phase space. Provided that the quench does not change the energy landscape too drastically, any trajectory starting from initial conditions inside a lake will remain confined to it. The system will be unable to explore the whole phase space and, consequently, it will not reach a state compatible with thermal equilibrium. The case (iii), in which the system never reaches a stationary state, is related to the existence of the so-called threshold level, a region in phase space in which the potential energy is dominated by saddles points. Dynamics within the threshold level are characterised by slow relaxation and ageing.

We determine the dynamic phase diagram as a function of the pre and post quench parameters, with dynamic transitions lines and phases that we characterise. The behaviour found is robust against the coupling to a bath.

The paper is organised as follows. In section 2 we introduce the model and we recall some of its main properties. Section 3 explains the quenches that we implement. In scetion 4 we present the analytic developments and asymptotic results and in section 5 we show the outcome of the numerical integration of the exact equations of motion. Section 6 presents the dynamic phase diagram. Finally, in section 7 we present our conclusions and we briefly discuss our future projects.

\section{Background}

\subsection{The Hamiltonian spherical $p$-spin model}

The $p$-spin model is a model with interactions between $p$ spins mediated by quenched random couplings $J_{i_{1} \ldots i_{p}}$. The potential energy is [8-12] 
Non equilibrium dynamics of isolated disordered systems: the classical Hamiltonian p-spin model

$$
H_{\text {pot }}\left[\left\{s_{i}\right\}\right]=-\frac{1}{p !} \sum_{i_{1} \neq \cdots \neq i_{p}}^{N} J_{i_{1} \ldots i_{p}} s_{i_{1}} \ldots s_{i_{p}} .
$$

The coupling exchanges are independent identically distributed random variables taken from a Gaussian distribution with average and variance

$$
\left[J_{i_{1} \ldots i_{p}}\right]-0, \quad\left[J_{i_{1}+\cdots+i_{p}}^{2}\right]=\frac{J^{2} p !}{2 N^{p-1}} .
$$

The parameter $J$ characterises the width of the Gaussian. In its standard spin-glass setting the spins are Ising variables. We will, instead, use continuous variables, $-\sqrt{N} \leqslant s_{i} \leqslant \sqrt{N}$ with $i=1, \ldots, N$, globally forced to satisfy (on averagc) a spherical constraint, $\sum_{i=1}^{N} s_{i}^{2}-N$, with $N$ the total number of spins [16]. The spherical constraint is imposed on average by adding a term

$$
H_{\text {constr }}-\frac{z}{2}\left(\sum s_{i}^{2}-N\right)
$$

to the Hamiltonian, with $z$ a Lagrange multiplier. The spins thus defined do not have an intrinsic dynamics. In statistical physics applications their temporal evolution is given by the coupling to a thermal bath, via a Monte Carlo rule or a Langevin equation [17].

The model can be endowed with conservative dynamics if one changes the 'spin' interpretation into a 'particle' one by adding a kinetic energy [18, 19]

$$
H_{\text {l:in }}\left[\left\{\dot{s}_{i}\right\}\right]=\frac{m}{2} \sum_{i=1}^{N}\left(\dot{s}_{i}\right)^{2}
$$

to the potential energy. The total energy of the Hamiltonian spherical p-spin model is then

$$
H_{\text {syst }}=H_{\text {kin }}+H_{\text {pot }}+H_{\text {constr }} .
$$

This model represents a particle constrained to move on an $N$-dimensional hypersphere with radius $\sqrt{N}$. The position of the particle is given by an N-dimensional vector $\vec{s}=\left(s_{1}, \ldots, s_{N}\right)$ and its velocity by another $N$-dimensional vector $\dot{\vec{s}}=\left(\dot{s}_{1}, \ldots, \dot{s}_{N}\right)$. The $N$ coordinates $s_{i}$ are globally constrained to lie, as a vector, on the hypersphere with radius $\sqrt{N}$. The velocity vector $\dot{\vec{s}}$ is, on average, perpendicular to $\vec{s}$, due to the spherical constraint. The parameter $m$ is the mass of the particle. The parameter $p$ is an integer and we will take it to be equal to 3 in the numerical applications.

The potential energy (1) is one instance of a generic random potential $V\left(\left\{s_{i}\right\}\right)$ with zero mean and corrclations [20-22]

$$
\left[V\left(\left\{s_{i}\right\}\right) V\left(\left\{s_{i}^{\prime}\right\}\right)\right]=-N \mathcal{V}\left(\left|\vec{s}-\vec{s}^{\prime}\right| / N\right)
$$

with $\mathcal{V}(|\vec{s}-\vec{s}| / N)--\frac{J^{2}}{2}(\vec{s} \cdot \vec{s} / N)^{p}$. The problem is also interesting for generic $\mathcal{V}$ but we will focus here on the monomial case that corresponds to the $p$-spin model. Details on the changes induced by other functions $\mathcal{V}$ will be given elsewhere.

The generic set of $N$ equations of motion for the system coupled to a white bath is

$$
m \ddot{s}_{i}(t)+\gamma \dot{s}_{i}(t)+z(t) s_{i}(t)-\sum_{\left(i_{2}<\cdots<i_{p}\right) \neq i} J_{i i_{2} \ldots i_{p}} s_{i_{2}}(t) \ldots s_{i_{p}}(t)+\xi_{i}(t)
$$


Non equilibrium dynamics of isolated disordered systems: the classical Hamiltonian p-spin model

where the random force $\xi_{i}$ has a Gaussian distribution with zero mean and correlations $\left\langle\xi_{i}(t) \xi_{j}\left(t^{\prime}\right)\right\rangle-2 \gamma T \delta\left(t-t^{\prime}\right), \gamma$ is the friction coefficient and $T$ is temperature. We have set, and we will keep, the Boltzmann constant to be $k_{\mathrm{B}}-1$. We have added the friction and noise terms to the Newton equation for completeness and to make contact with the stochastic setting usually used in the study of this model. Still, having introduced in equation (4) the kinetic energy that provides an intrinsic dynamics to the system, we will be able to switch off the coupling to the bath, that is to say set $\gamma=0$, and study the dynamics of the isolated system.

The initial condition will be taken to be $\left\{s_{i}^{0}, \dot{s}_{i}^{0}\right\} \equiv\left\{s_{i}(0), \dot{s}_{i}(0)\right\}$ and chosen in ways that we specify below.

\subsection{The $p \geqslant 3$ case}

The spherical $p$-spin model bchaves very differently for $p=2$ and $p \geqslant 3$. In particular, for $p \geqslant 3$ the dynamics are non-linear, whereas the dynamics of the $p=2$ spin model can be recast, through the diagonalization of the interaction matrix, into the dynamics of harmonic oscillators coupled only through the Lagrange multiplier that enforces the spherical constraint. Moreover, as we shall describe in detail below, the potential energy landscape for $p \geqslant 3$ is complex, with an extensive number of local minima with a non-trivial effect on the dynamics. For $p-2$ the energy landscape possesses only one absolute minimum which dominates the low energy dynamics. In the present work we concentrate on the cases $p \geqslant 3$ and we leave for a future study the $p=2$ model.

The model with $p \geqslant 3$ has a very rich and complex free-energy landscape with interesting metastability. In the past, the model with just the potential energy was analysed in a considerable degree of detail. The kinetic energy allows one to study the dynamics of the isolated system without changing the picture of metastability described below, since it contributes a trivial Maxwell (Gaussian) factor to the canonical probability weight.

In short, the known results for the spherical $p \geqslant 3$ model can be summarised as follows. In the thermodynamic limit, $N \rightarrow \infty$, the model has two 'critical' temperatures $T_{s}<T_{d}$. At high temperatures, $T>T_{d}$, with $T_{d}$ the dynamical critical temperature, the equilibrium state is the paramagnetic one, with vanishing local order parameters. Below $T_{d}$, there is an exponentially large number of metastable states. For temperatures between $T_{s}$ and $T_{d}$ the equilibrium state remains paramagnetic. However, below $T_{d}$ the dynamics is stuck into the highest lying metastable states (the threshold level) and departs from the paramagnetic dynamics observed above $T_{d}$. In particular, for quenches from above to below $T_{d}$ the relaxation is non-stationary with ageing effects and other special features due to the fact that it occurs, asymptotically, on a flat region of phase space. Finally, below $T_{s}$ the equilibrium state is no longer paramagnetic, there is replica symmetry breaking.

The analysis of the order-parameter dependent free-energy landscape proves that there is an exponentially large number of metastable states above the static critical temperature $T_{s}$, (and until some high temperature $T_{\text {max }}$ ). Between $T_{d}$ and $T_{s}$ equilibrium is dominated by a class of metastable states that exist in exponentially large number (finite complexity). Below $T_{s}$ the number of metastable states is no longer exponential and the Gibbs-Boltzmann measure is dominated by the low lying ones. The dissipative relaxation dynamics of the model is consistent with the picture emerging 
from the static analysis. It is also surprising since for quenches from above to below $T_{d}$ the relaxation is non-stationary with ageing effects and other special features due to the fact that it occurs, asymptotically, on a flat region of phase space.

In the rest of this section we turn the description in the previous paragraph quantitatively. We give some details on how to derive the picture above with three methods: the replica trick, the Thouless-Anderson-Palmer (TAP) approach and the dissipative dynamics. We give the expressions for some characteristic temperatures and energies that will be useful in the study of the quench dynamics of the isolated system and we sum up, in a table, the values these take in the $p-3$ case.

2.2.1. Equilibrium. The static properties of a model with quenched randomness follow from the study of the disorder-averaged free-energy density in the canonical ensemble. The kinetic energy term in (4) is a conventional one and it does not depend on the quenched disordered interactions. The statistical sum over the velocities in the partition function can be readily performed and it yields the usual factor stemming from the Maxwell weight, $[2 \pi /(\beta m)]^{N / 2}$. Instead, the contribution of the potential energy to the average over disorder of the logarithm of the partition function has been computed with the help of the replica trick [16]. In this framework one introduces an $n \times n$ 'overlap' matrix $Q_{a b}-N^{-1} \sum_{i=1}^{N}\left[\left\langle s_{i}^{a} s_{i}^{b}\right\rangle\right]$ between replicas $a$ and $b(a, b=1, \ldots n)$ where the angular brackets denote the statistical average. In the case of the $p \geqslant 3$ model, the one step replica symmetric Ansatz solves this problem exactly below $T_{s}$ in the $N \rightarrow \infty$ limit. Within this Ansatz the matrix is parametrized as $Q_{a b}=\delta_{a b}+q_{\mathrm{ea}} \epsilon_{a b}$, with $\epsilon_{a b}$ equal to one if $a b$ are within a diagonal square block of size $m_{\mathrm{eq}} \times m_{\mathrm{eq}}$ and zero otherwise. The parameters $m_{e q}$ and $q_{e a}$ are determined by requiring that they extremise the free-energy density calculated in the limit $n \rightarrow 0$ (after $N \rightarrow \infty$ ).

The Edwards-Anderson parameter, $q_{\mathrm{e}}$, and the replica parameter, $m_{\mathrm{eq}}$ were derived in [16]. They are given, in implicit form, by the equations

$$
\begin{aligned}
& \frac{1}{p}=-y \frac{1-y+\ln y}{(1-y)^{2}}, \\
& \frac{p}{2} q_{\mathrm{ea}}^{p-2}\left(1-q_{\mathrm{ea}}\right)^{2}=y \frac{T^{2}}{J^{2}}, \\
& m_{\mathrm{eq}}=\frac{1-y}{y} \frac{1-q_{\mathrm{ea}}}{q_{\mathrm{ea}}},
\end{aligned}
$$

that are easy to solve numerically. In the $T \rightarrow 0$ limit, $q_{\mathrm{ea}}-1-a T$ and $m_{\mathrm{eq}}-b T$ with $a=(2 y / p)^{1 / 2}$ and $b=a(1-y) / y$.

The static transition temperature occurs at $T_{s}$ such that $m_{\mathrm{eq}}=1$. This condition implies

$$
q_{\mathrm{s}}=q_{\mathrm{ea}}\left(T_{s}^{\prime}\right)=1-y
$$

that in turn fixes $T_{s}$ to $[16,23,24]$

$$
T_{s}=\sqrt{\frac{p y}{2}}(1-y)^{\frac{p}{2}-1} J .
$$


Specializing to the $p-3$ case, $T_{s}^{\prime}=0.586 \mathrm{~J}$. The phase transition is discontinuous, in the sense that the order parameter $q_{\mathrm{ca}}$ jumps at $T_{s}, \lim _{T \rightarrow T_{s}^{-}} q_{\mathrm{ea}}(T) \neq 0$ and $\lim _{T \rightarrow T_{j}^{-}} m_{\text {ey }}(T)-1$ while above $T_{s}, q_{\text {ea }}(T)=0$. The solution boils down, at high temperature, to a replica symmetric one. However, there are no jumps in the thermodynamic quantities so the transition is not of the first order in this sense. It is a 'random first order phase transition'.

The cquilibrium potential encrgy density, $e_{\text {pot }}^{\mathrm{eq}} \equiv\left\langle H_{\text {pot }}\right\rangle$, is then

$$
e_{\text {pot }}^{\mathrm{eq}}(I)=-\frac{J^{2}}{2 T}\left[1-\left(1-m_{\mathrm{eq}}\right) q_{\mathrm{ea}}^{p}\right]
$$

that simplifies to $e_{\text {pot }}^{\text {eq }}=-J^{2} /(2 T)$ above $T_{s}$ and takes the value $e_{\text {pot }}^{\text {eq }}\left(T_{s}\right)=-0.853 . J$ at $T_{s}$ for $p=3$. At zero temperature the equilibrium potential energy is

$$
e_{\text {pot }}^{\mathrm{eq}}(T \rightarrow 0)=-\frac{J}{\sqrt{2 p y}}|1+(p-1) y|
$$

and $c_{\text {pot }}^{\text {eq }}=-1.172 J$ at $T=0$ for $p=3$.

Above $T_{s}$ the qea $\neq 0$ solution still exists (with $m_{\mathrm{eq}}>1$ ) and it disappears at a much higher temperature, $T_{\mathrm{eq}}^{\max }$, where

$$
\underset{\text { पea }}{\max }=\frac{p-2}{p} \quad \text { and } \quad T_{\mathrm{eq}}^{\max }=\sqrt{\frac{2}{y p}}\left(\frac{p-2}{p}\right)^{(p-2) / 2} . J .
$$

For $p=3, T_{\mathrm{eq}}^{\max }=0.791 \mathrm{~J}$.

2.2.2. Metastability. The Thouless-Anderson-Palmer (TAP) method serves to derive mean-ficld cquations for the local order parameters, the local magnetisations,

$$
m_{i}-\left\langle s_{i}\right\rangle
$$

at fixed quenched disorder in the canonical ensemble. As everywhere in the paper, the angular brackets denote statistical average.

The TAP method also constructs the full free-energy and potential encrgy density landscapes. It has been applied to the study of the disordered p-spin models landscapes in, e.g. [25-28]. The outcome for the potential energy as a function of temperature is summarised in figure 1. We explain the meaning of the different lines, and the special values of the potential energy and temperature highlighted in the figure, in the rest of this section.

First of all, it is convenient to introduce

$$
q \equiv \frac{1}{N} \sum_{i=1}^{N} m_{i}^{2}
$$

and then observe that, for the spherical $p$-spin model [27],

$$
\left.H_{\text {pot }} \mid\left\{m h_{i}\right\}\right]=q^{p / 2} H_{\text {pot }}\left[\left\{s_{i}\right\}\right],
$$

due to the homogeneity of the potential energy. This property is not general and makes the structure of the potential energy landscape of the spherical $p$-spin model particularly simple, with no level crossings nor birth of states at finite temperature, as 


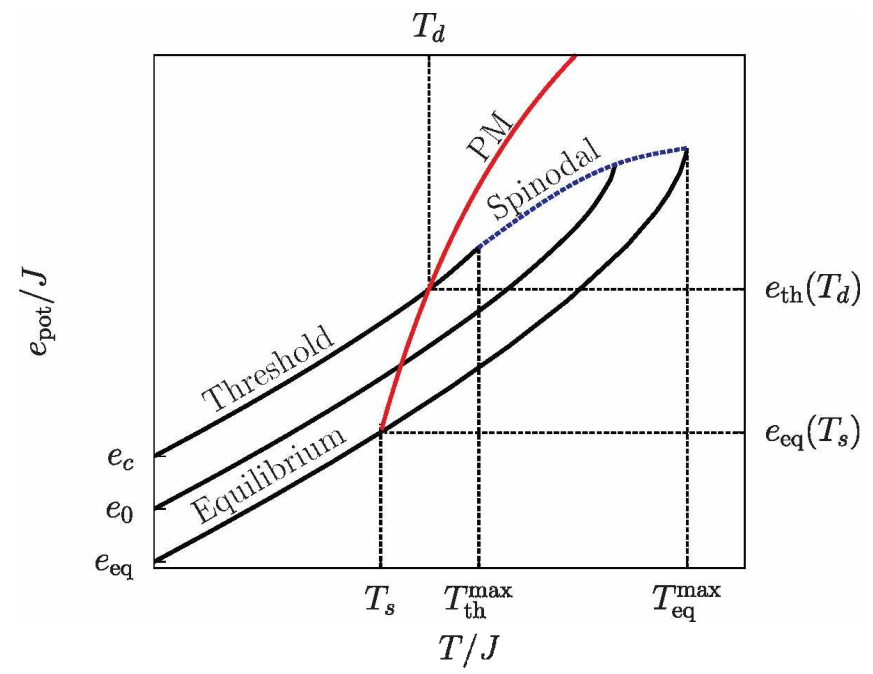

\begin{tabular}{|c|c|c|c|c|c|}
\hline & $T=0$ & $T_{s}=0.586$ & $T_{d}=0.6123$ & $T_{\mathrm{th}}^{\max }=2 / 3$ & $T_{\mathrm{eq}}^{\text {max }}=0.7912$ \\
\hline \hline$e_{\mathrm{th}}$ & -1.155 & -0.853 & -0.839 & -0.778 & \\
\hline$q_{\mathrm{th}}$ & 1 & 0.539 & 0.500 & $1 / 3$ & \\
\hline$e_{\mathrm{eq}}$ & -1.172 & -0.853 & -0.817 & -0.750 & -0.632 \\
\hline$q_{\mathrm{ea}}$ & 1 & 0.645 & 0.622 & 0.570 & $1 / 3$ \\
\hline
\end{tabular}

Figure 1. Sketch of the potential energy $e_{\text {pot }}$ as a function of temperature $T$. The lines represent the temperature dependence of the potential energy for some selected states (the equilibrium state, the threshold state and an intermediate state). We also show the paramagnetic energy (with a full red line) and the spinodal line (with a dotted blue line). We mark on the plot all the relevant energies and temperatures. Notice that the potential energy is negative. In the table we show some typical values of temperatures and energies in units of $J$ for the $p-3$ spherical model.

sketched in figure 1. In other words, each state can be univocally labeled by its zerotemperature potential energy.

The extremisation conditions on the TAP free-energy lead to [27]

$$
(p-1)(1-q) q^{(p-2) / 2}=\frac{T}{J^{2}}\left(-e_{0}-\sqrt{e_{0}^{2}-e_{c}^{2}}\right)
$$

with

$$
e_{c}=-\sqrt{\frac{2(p-1)}{p}} J,
$$

and $e_{0}$ the zero temperature energy. This equation admits real solutions for $e_{0}$ such that $e_{\mathrm{eq}}(T-0)<e_{0}<e_{c}<0$, with $e_{c}$ the threshold energy at zero temperature. Each of these solutions is an equilibrium or metastable state of the system. Equation (19) 
determines $q$ as a function of the energy density of a TAP solution at $T-0$, en, and temperature, $T$, both measured in units of $J$. In particular, one can check that replacing $c_{0}$ by $e_{\text {eq }}(T-0)$ as given by equation (14), the cquation fixing the equilibrium Edwards-Anderson parameter, equation (9), is recovered. Otherwise, replacing $e_{0}$ by $e_{c}$ the equation for the threshold $q_{\mathrm{th}}$ is obtained. The left-hand-side (lhs) has a bell-shaped form with a maximum at $q_{\max }=(p-2) / p$. For $q=q_{\max }$ the same equation determines $T_{\max }\left(e_{0}\right)$ above which the cquation has no more solution. The physical solution is the one taking the largest value of $q$, since it continuously connects to the zero-temperature one in which $q-1$. Different TAP states cease to exist at different temperatures, as sketched in figure 1. The temperature at which this occurs for the equilibrium level is given in equation (15). The threshold level, instead, disappears at

$$
T_{\mathrm{th}}^{\max }=\sqrt{\frac{2}{p}(p-1)}\left(\frac{p-2}{p}\right)^{\frac{p-2}{2}} .7
$$

and $T_{\mathrm{th}}^{\max }-2 / 3 . J$ for $p=3$.

An expression of the potential energy $e$ as a function of $e_{0}$ and $q$ (itself a function of $T$ ) is

$$
e\left(T, e_{0}\right)=q^{p / 2} e_{0}-\frac{J^{2}}{2 T}\left[(p-1) q^{p}-p q^{p-1}+1\right] .
$$

Of particular interest is the threshold potential energy that we will give explicitly in equation (32).

The equation that fixes the $q$ values of the TAP states that combine (because of their macroscopic degeneracy) to yield equilibrium in the range $T_{s}<T<T_{d}$ is $[23,27]$

$$
\frac{p}{2} q^{p-2}(1-q)-\frac{T^{2}}{J^{2}} .
$$

(These states have thermodynamic properties, like the internal energy, that coincide with the continuation of the high-temperature paramagnetic one. In the sketch in figure 1 these states correspond to points along the continuation of the PM line (red) inside the region where metastable states exist, i.c. the portion of the PM curve between $T_{s}$ and $T_{d \cdot}$. Once again, the bell-shape form of the lhs of equation (23) implies that this equation admits a solution $q \neq 0$ for temperatures such that $T<T^{\text {max }}$ with

$$
\left(T^{\max }\right)^{2}=a^{2}(p) \cdot J^{2}
$$

were we have introduced the numerical constant

$$
a^{2}(p)=\frac{p}{2} \frac{(p-2)^{p-2}}{(p-1)^{p-1}}
$$

that is smaller than 1 for all $p \geqslant 3$.

We will recall the dynamic significance of these TAP states in the next section. We announce here that $T^{\max }$ and $q\left(T^{\max }\right)$ coincide with $T_{d}$ and $q_{d}$, the critical dynamical temperature and the value of the parameter $q$ at this temperature. In particular, for $p-3, T^{\max }=\sqrt{3 / 8} J \simeq 0.612 J=T_{d}$ and at this temperature, $q=q_{\max }=1 / 2$. 
Non equilibrium dynamics of isolated disordered systems: the classical Hamiltonian p-spin model

The scenario with a large multiplicity of metastable states has been confirmed with the exhaustive cnumeration of the extrema of the TAP potential energy landscape of finite (small) size spherical $p$-spin models at fixed randomness [29].

2.2.3. Relaxation dynamics. The over-damped relaxation dynamics of the spherical $p$-spin model (coupled to a Markovian bath) were studied in $[17,30,31]$. The dynamics considered in these papers evolve a completely random initial condition, $\left\{s_{i}^{0}\right\}$, that, for purely relaxational dynamics corresponds (formally) to an infinite temperature initial state. The latter is then quenched to a final state in contact with a bath at finite temperature $T$. The analysis is performed in the thermodynamic limit, $N \rightarrow \infty$, and times are taken to infinity only after, remaining therefore finite with respect to $N$.

For quenches with $T>T_{d}$ the dynamics quickly approach equilibrium at the new temperature. The correlation and linear response are invariant under translations of time and they are related by the fluctuation dissipation theorem.

Above but close to $T_{d}$ the relaxation exhibits a strong slowing down with the correlation decaying in two steps, with a first approach to a plateaul and a further decay from this plateau, in a much longer time-scale, to zero. This is similar to what is observed in super-cooled liquids and it is the reason why this model has been used as a toy model for glass formers of fragile kind.

For $T<T_{d}$, the evolution of the correlation and linear response functions conform to the weak-egodicity breaking scenario $[17,31]$ in which they separate in two contributions evolving in different two-time regimes

$$
\begin{aligned}
& C\left(t_{1}, t_{2}\right)=C_{\mathrm{st}}\left(t_{1}-t_{2}\right)+C_{\mathrm{ag}}\left(t_{1}, t_{2}\right) . \\
& R\left(t_{1}, t_{2}\right)-R_{\mathrm{st}}\left(t_{1}-t_{2}\right)+R_{\mathrm{ag}}\left(t_{1}, t_{2}\right),
\end{aligned}
$$

with the stationary and a non-stationary terms linked by the fluctuation-dissipation theorem (FDT) at the temperature of the bath and a modified FDT at an effective temperature $T_{\text {eff }}[32,33]$ selected by the dynamics,

$$
\begin{aligned}
& R_{\mathrm{st}}\left(t_{1}-t_{2}\right)=-\frac{1}{T} \frac{d C_{\mathrm{st}}\left(t_{1}-t_{2}\right)}{d\left(t_{1}-t_{2}\right)}, \\
& R_{\mathrm{ag}}\left(t_{1}-t_{2}\right)=\frac{1}{T_{\mathrm{eff}}} \frac{\partial C_{\mathrm{ag}}\left(t_{1}, t_{2}\right)}{\partial t_{2}},
\end{aligned}
$$

always with $t_{1} \geqslant \iota_{2}$. In the asymptotic limit, the two terms added to form $C$ and $R$ evolve in different regimes in the sense that when one changes the other one is constant and vice versa. The limiting values of the various contributions to the correlation function are

$$
\begin{array}{lc}
C_{\mathrm{st}}(0)=1-q, & \lim _{\tau \rightarrow \infty} C_{\mathrm{st}}(\tau)=0, \\
\lim _{t_{2} \rightarrow t_{1}^{-}} C_{\mathrm{ag}}\left(t_{1}, t_{2}\right)-q, & \lim _{t_{1} \gg t_{2}} C_{\mathrm{ag}}\left(t_{1}, t_{2}\right)-0,
\end{array}
$$

with $q$ being equal to $q_{\text {th }}$, the value at the threshold of the TAP free-energy density. 
The asymptotic potential energy reached after the quench for $T<T_{d}$ is the one of the threshold level in the free-cnergy landscape:

$$
e_{\mathrm{th}}=-\frac{J^{2}}{2}\left[\frac{1}{T}\left(1-q_{\mathrm{th}}^{p}\right)+\frac{1}{T_{\mathrm{eff}}} q_{\mathrm{th}}^{p}\right] \text {. }
$$

This expression will be very useful in the numerical analysis. The parameters $q_{\mathrm{th}}$ and $T_{\text {eff }}$ are given by

$$
\begin{aligned}
& \frac{p(p-1)}{2}\left(1-q_{\mathrm{th}}\right)^{2} q_{\mathrm{th}}^{p-2}=\frac{T^{2}}{J^{2}}, \\
& m_{\mathrm{th}} \equiv \frac{T}{T_{\mathrm{eff}}}=\frac{(p-2)\left(1-q_{\mathrm{hh}}\right)}{q_{\mathrm{th}}} .
\end{aligned}
$$

The parameter $m_{\mathrm{th}}$ measures the violation of the fluctuation-dissipation theorem out of equilibrium and can be interpreted in terms of an effective temperature $T_{\text {eff }}$ [32] (note that $m_{\text {th }} \leqslant 1$ and $T_{\text {eff }}>T$ ' for quenches from high to low temperature).

The bell-shaped function in the lhs of equation (33) indicates, once again, that this equation has two solutions until a temperature $T_{\mathrm{th}}^{\max }$ given by the same expression, equation (21), obtained with the TAP formalism. The relevant solution is the one taking the higher value, the one that is connected to $q_{\mathrm{th}}-1$ at $T-0$. Its value and the energy at this temperature are given in the table that accompanies figure 1.

The equation fixing $q_{\text {th }}$ as a function of $T$ is implicit so we cannot write an explicit expression for $e_{\mathrm{th}}(T)$. We can, instead, eliminate $T$ from equation (32) using equations (33) and (34), and then recast equation (32) as

$$
e_{\mathrm{th}}=-\frac{J}{\sqrt{2 p(p-1)}} q_{\mathrm{th}}^{(2-p) / 2}\left[\frac{1-q_{\mathrm{th}}^{p}}{1-q_{\mathrm{th}}}+(p-2) q_{\mathrm{th}}^{p-1}\right] .
$$

At $T \simeq 0, q_{\mathrm{th}} \simeq 1-a T$ with $a^{2}=2 /(p(p-1))$ and $m_{\mathrm{th}} \simeq(p-2) a T$. Expanding in powers of $T$ one finds $e_{\mathrm{th}} \simeq-a(p-1) / 2$ and then

$$
e_{\mathrm{th}}(T=0)=-\sqrt{\frac{2(p-1)}{p}} J \equiv e_{c}
$$

as cxpected. Its concrete value for $p=3$ is given in table.

The dynamic critical temperature arises when $m_{\mathrm{th}}=1$ :

$$
T_{d}-a(p) J
$$

that coincides with equation (24). Specialising to the $p-3$ case $T_{d}-0.612 J$. When this occurs $e_{\mathrm{th}}\left(T_{d}\right)=e_{\mathrm{eq}}\left(T_{d}\right)=-J^{2} /\left(2 T_{d}\right)$ and $q=q_{d}=q_{\mathrm{th}}\left(T_{d}\right)=(p-2) /(p-1)$.

The dynamic relevance of the TAP states that are non trivial but 'confused' with the paramagnetic solution with the conventional replica calculation, the ones with $q$ values determined by equation (23), is understood from the analysis of the relaxation dynamics starting from initial conditions in equilibrium at the range of temperature $T_{s}<T^{\prime \prime}<T_{d}$ [34-37]. These initial conditions are confined within TAP states that do not let the system escape. The asymptotic dynamics remain within the departing state, as indicated by the fact that $\lim _{t_{1} \rightarrow \infty} \lim _{l_{2} \rightarrow \infty} C\left(t_{1}, t_{2}\right)-q$, with $q$ given by equation (23). 
Non equilibrium dynamics of isolated disordered systems: the classical Hamiltonian p-spin model

For finite $N$ the threshold level dynamics and evolution within TAP states should have finite, though exponentially large in $N$, lifetime. Some numerical evidence for this was given in, e.g. $\lceil 38-40\rceil$.

\section{Quenches and dynamics of the isolated system}

The Hamiltonian (5) with $H_{\text {kin }}$ and $H_{\text {pot }}$ given in equations (4) and (1) has two parameters, the variance of the couplings $J_{i_{1} \ldots i_{p}}$ and the mass of the particle. We will consider initial conditions sampled from equilibrium at $T^{\prime}$ with Hamiltonian $H_{0}$ and evolve them with a different Hamiltonian $H$. Since the potential and kinetic energies play different roles, as the former depends on the quenched randomness while the latter does not, the treatment of the quenches induced by a change in the random interactions needs a bit more care.

Let us take the $p$-spin model (1) and (4) with coupling constants $J_{i_{1} \ldots i_{p}}^{0}$ in canonical equilibrium at temperature $T^{\prime}$ and evolve it in isolation from the environment with a modified Hamiltonian. Quenches in the random exchanges that do not keep any memory of the values before the quench are not interesting. We therefore impose quenches in the potential energy such that each random choice of the exchanges is changed into $J_{i_{1} \ldots i_{p}}$, with the new couplings related to the old ones by

$$
J_{i_{1} \ldots i_{p}}^{0}=J_{i_{1} \ldots i_{p}} x \quad \forall i_{1}, \ldots, i_{p} .
$$

This transformation is such that for each sample (disorder realisation) at $t=0$ we uniformly change the value of all random couplings by the same factor. In other words, we prepare the system in a thermal state of a Hamiltonian with potential energy

$$
H_{\text {pot }}^{0}\left[\left\{s_{i}\right\}\right]--\sum_{1 \leqslant i_{1}<\ldots<i_{p} \leqslant N} J_{i_{1} \ldots i_{p}}^{0} s_{i_{1}} \ldots s_{i_{p}},
$$

but let each initial condition sampled from this state evolve with the Hamiltonian with potential energy

$$
H_{\text {pot }}\left[\left\{s_{i}\right\}\right]=-\sum_{1 \leqslant i_{1}<\ldots<i_{p} \leqslant N} J_{i_{1} \ldots i_{p}} s_{i_{1}} \ldots s_{i_{p}} .
$$

Note that with $x<1$ we enhance the interactions and with $x>1$ we depress the interactions between the spins. Technically, after relating the coupling strengths one by one we have only one quenched disorder average to make.

It is important to note that under this change, the potential energy levels in figure 1 are translated upwards or downwards, and stretched or contracted, for $x<1$ or $x>1$, respectively. Indeed, one can easily see the translation by noticing that $\epsilon_{0}$ is proportional to $J$, and the contraction by noticing that the various $T_{\text {max }}$ are proportional to $J$. Concomitantly, the static and dynamic critical temperatures $T_{s}^{0}=T_{s}\left(J_{0}\right)$ and $T_{d}^{0}-T_{d}\left(J_{0}\right)$ of the initial potential are shifted to new values $T_{s}-T_{s}(J)$ and $T_{d}-T_{d}(J)$ after the quench,

$$
\frac{T_{s}}{T_{s}^{0}}-\frac{J}{J_{0}}-\frac{1}{x} \quad \Rightarrow \quad T_{s}^{0}-x T_{s} \quad \text { and } \quad T_{d}^{0}-x T_{d} .
$$


Non equilibrium dynamics of isolated disordered systems: the classical Hamiltonian p-spin model

We will also consider quenches in the mass, $m_{0} \mapsto m$, that change the kinetic contribution to the energy as

$$
H_{\mathrm{kin}}^{0}=\frac{m_{0}}{2} \sum_{i} \dot{s}_{i}^{2} \quad \mapsto \quad H_{\mathrm{kin}}=\frac{m}{2} \sum_{i} \dot{s}_{i}^{2} .
$$

\subsection{Dynamical equations}

Importantly cnough, all our results will be derived after having taken the limit $N \rightarrow \infty$ from the start, and eventually considering the long-times asymptotic limit only after.

In the limit $N \rightarrow \infty$ the dynamics of the model are fully characterised by the behaviour of the two-time correlation and linear response function. The equations ruling their evolution are easily derived using the Martin-Siggia-Rose functional formalism.

Particular initial conditions can be imposed by including in the dynamic generating function an integration over the initial conditions weighted with their distribution. Equilibrium initial conditions at a temperature $T^{\prime}-\beta^{\prime-1}$ are distributed according to the Gibbs-Boltzmann measure

$$
P\left(\left\{s_{i}(0), \dot{s}_{i}(0)\right\}\right)-Z^{-1}\left(\beta^{\prime}\right) \mathrm{e}^{-\beta^{\prime} H_{0}\left(\left\{s_{i}(0), \dot{s}_{i}(0)\right)\right\}}
$$

with $H_{0}$ defined in equations (1)-(5). The Hamiltonian depends on the quenched random interactions. The average over disorder in the case of initial states correlated with the quenched randomness needs the use of the replica trick, as explained in [34]. This means that the spin variables evaluated at the initial time have to be replicated, $s_{i}(0) \mapsto s_{i}^{a}(0)$, with $a-1, \ldots, n$, to perform the average over the random exchanges. The subsequent evolution of each of these replicas has to be followed in time, and it turns out that the replica structure of the initial condition is conserved.

For the dissipative spherical $p$-spin model this calculation has been carried out in $[36,37$, and it can be adapted to the isolated model with kinctic energy with just minor modifications. We therefore present the outcome here without giving many details of the derivation. In order to facilitate the comparison to the expressions for the dissipative model we keep the coupling to the white bath active in the presentation of the dynamic equations. Later on, we will focus on the isolated problem.

In the $N \rightarrow \infty$ limit, the only relevant correlation and linear response functions that determine the dynamics of the model are

$$
\begin{aligned}
& \left.C_{a b}\left(t_{1}, t_{2}\right)=N^{-1} \sum_{i=1}^{N} \mid\left\langle s_{i}^{a}\left(t_{1}\right) s_{i}^{b}\left(t_{2}\right)\right\rangle\right] . \\
& C_{a b}\left(t_{1}, 0\right)=N^{-1} \sum_{i=1}^{N}\left[\left\langle s_{i}^{a}\left(t_{1}\right) s_{i}^{b}(0)\right\rangle\right], \\
& R_{a b}\left(t_{1}, l_{2}\right)=\left.N^{-1} \frac{\delta}{\delta h_{b}\left(t_{2}\right)} \sum_{i=1}^{N}\left[\left\langle s_{i}^{a(h)}\left(\iota_{1}\right)\right\rangle\right]\right|_{h=0},
\end{aligned}
$$


Non equilibrium dynamics of isolated disordered systems: the classical Hamiltonian p-spin model

for $t_{1}, t_{2}>0$, where the infinitesimal perturbation $h$ is coupled linearly to the spin $H \mapsto H-h \sum_{i=1}^{N} s_{i}$ at time $t_{2}$ and the upperscript $(h)$ indicates that the configuration is measured after having applied the field $h$. The square brackets denote here and everywhere in the paper the average over quenched disorder. The angular brackets indicate the average over thermal noise if the system is coupled to an environment, and over the initial conditions of the dynamics sampled with the probability distribution $P$. When the coupling to the bath is set to zero, $\gamma=0$, the last average is the only one remaining in the angular brackets operation.

Without loss of generality we will focus on initial states in equilibrium at $T^{\prime} \geqslant T_{s}^{0}$, where the replica structure is symmetric, although there can still be a complex structure of metastable states, as explained in section 2.2.2. Considering the case $T^{\prime}<T_{s}^{0}$ would add quite a lot of unnecessary complexity to the calculations, while we do not expect major changes in the dynamic behaviour under such initial conditions. For these reasons, the following expressions are valid only for $T^{\prime} \geqslant T_{s}^{0}$.

The dynamical equations of the model coupled to a bath at temperature $T$, starting from a random state, are well known and can be found in Refs. [17, 23, 24, 31]. They can be derived from the dynamical Martin-Siggia-Rose action

$$
S_{J}=\sum_{i=1}^{N} \int_{0}^{\infty} \mathrm{d} t \dot{s}_{i}(t)\left(\gamma T \hat{s}_{i}(t)+\gamma \dot{s}_{i}(t)+m \ddot{s}_{i}(t)+\frac{\partial H_{\mathrm{pot}}}{\partial s_{i}}+z(t) s_{i}(t)\right),
$$

where the subscript $J$ in $S_{J}$ indicates that the action depends explicitly on the disordered couplings and the $\hat{s}_{i}$ are (imaginary) auxiliary variables used to rewrite a delta function that enforces the validity of the equation of motion in the path integral. (This form corresponds to the Ito convention in which there is no Jacobian contribution.) We included here the kinetic energy contribution not present in these publications.

The replicated dynamical action that includes the contribution from the distribution of the initial conditions reads

$S_{J}-\sum_{a=1}^{n} \sum_{i=1}^{N} \int_{j}^{\infty} \mathrm{d} t \hat{s}_{i}^{a}(t)\left(\gamma T \hat{s}_{\bar{x}}^{\prime \prime}(t)+\gamma \dot{s}_{i}^{\prime}(t)+m \dot{s}_{i}^{u}(t)+\frac{\partial H_{\mathrm{pot}}}{\partial s_{i}^{a}}+z(t) s_{i}^{u}(t)\right)-\frac{1}{T^{\prime}} H_{0}\left|s_{i}^{u}(0) ; \dot{s}_{i}^{a}(0)\right|$

The kinetic energy term in the initial distribution does not depend on the quenched random interactions, it does not affect the dynamic equations, but will appear only in the energetic considerations that we will develop below.

We will now show how to perform the average over the couplings in some detail. Two terms in (46) depend on the interactions, they are the ones in which $H_{\text {pot }}$ appears in the force in the evolution equation and in the initial contribution $H_{0}$. We collect them in $S_{\text {dis. }}$ (Note that we use, as a working assumption, that $z(l)$ does not depend on $J_{i j k}$.) The average over disorder of the exponentials of these two terms is

$$
\begin{aligned}
{\left[\mathrm{e}^{S_{\mathrm{dis}}}\right]=\prod_{i<k<l} \int \mathrm{d} J_{i k l} \exp \{} & -\frac{J_{i k l}^{2} 2 N^{p-1}}{2 p ! J^{2}}-\frac{1}{T^{\prime}} J_{i k l}^{0} \sum_{a} s_{i}^{a}(0) s_{k}^{a}(0) s_{l}^{a}(0) \\
& \left.-J_{i k l} \sum_{a} \int_{0}^{\infty} \mathrm{d} t\left(\hat{s}_{i}^{a}(t) s_{k}^{a}(t) s_{l}^{a}(t)+s_{i}^{a}(t) \hat{s}_{k}^{a}(t) s_{l}^{a}(t)+s_{i}^{a}(t) s_{k}^{a}(t) \hat{s}_{l}^{a}(t)\right)\right\},
\end{aligned}
$$


where we have included the Gaussian distribution over the couplings (we average over the final couplings $J_{i k l}$ but the same results would be obtained had we chosen to average over the initial ones, $\left.J_{i k l}^{0}\right)$. We have symmetrised the term originating in $\partial H_{\text {pot }} / \partial s_{i}^{a}$. Following our choice of quench we set $J_{i k l}^{0}=x J_{i k l} \forall\{i, k, l\}$, in which case the disorder dependent part of the action becomes

$$
\begin{aligned}
{\left[\mathrm{e}^{S_{\mathrm{dis}}}\right]=\prod_{i<k<l} \int \mathrm{d} J_{i k l} \exp } & \left\{-\frac{J_{i k l}^{2} 2 N^{p-1}}{2 p ! J^{2}}-\frac{x}{T} J_{i k l} \sum_{a} s_{i}^{a}(0) s_{k}^{a}(0) s_{l}^{a}(0)\right. \\
& \left.-J_{i k l} \sum_{a} \int_{0}^{\infty} \mathrm{d} t\left(\hat{s}_{i}^{a}(t) s_{k}^{a}(t) s_{l}^{a}(t)+s_{i}^{a}(t) \hat{s}_{k}^{a}(t) s_{l}^{a}(t)+s_{i}^{a}(t) s_{k}^{a}(t) \hat{s}_{l}^{a}(t)\right)\right\} .
\end{aligned}
$$

After performing the Gaussian integration over the couplings we have

$$
\begin{aligned}
{\left[e^{s_{\mathrm{di}}}\right] \times \prod_{i<k<l} \exp \left\{\frac { p ! J ^ { 2 } } { 4 N ^ { p - 1 } } \left[\int_{0}^{\infty} \mathrm{d} t\right.\right.} & \sum_{a}\left(s_{i}^{a}(t) s_{k}^{a}(t) s_{l}^{a}(t)+s_{i}^{a}(t) \hat{s}_{k}^{a}(t) s_{l}^{a}(t)+s_{i}^{a}(t) s_{k}^{a}(t) \hat{s}_{l}^{a}(t)\right) \\
& \left.+\frac{x}{T^{\prime}} \sum_{a} s_{i}^{a}(0) s_{k}^{a}(0) s_{l}^{a}(0)\right] \\
\times & {\left[\int_{0}^{\infty} \mathrm{d} t^{\prime} \sum_{b}\left(s_{i}^{b}\left(t^{\prime}\right) s_{k}^{b}\left(t^{\prime}\right) s_{l}^{b}\left(t^{\prime}\right)+s_{i}^{b}\left(t^{\prime}\right) \hat{s}_{k}^{b}\left(t^{\prime}\right) s_{l}^{b}\left(t^{\prime}\right) \mid s_{i}^{b}\left(t^{\prime}\right) s_{k}^{b}\left(t^{\prime}\right) \hat{s}_{l}^{b}\left(t^{\prime}\right)\right)\right.} \\
& \left.\left.+\frac{x}{T^{\prime}} \sum_{b} s_{i}^{b}(0) s_{k}^{b}(0) s_{l}^{b}(0)\right]\right\} .
\end{aligned}
$$

The product between the two terms involving integrals in time produces the terms already present in the dynamical equations starting from a random initial condition. These terms are proportional to $J^{2}$. The product between a term with one time integral and one term with the initial condition generates the new terms. They are proportional to $J^{2} x$, that is to say $J J_{0}$, if we call $J_{0}=x J$. The product of the terms involving only the initial conditions yields the equilibrium equations of the model decoupled from the dynamics, and are proportional to $J_{0}^{2}$, as they should.

Taking now $N \rightarrow \infty$ one derives the dynamical equations that read

$$
\begin{gathered}
\left(m \partial_{t_{1}}^{2}+\gamma \partial_{t_{1}}+z\left(t_{1}\right)\right) R\left(t_{1}, t_{2}\right)=\frac{J^{2} p(p-1)}{2} \int_{t_{2}}^{t_{1}} \mathrm{~d} t^{\prime} R\left(t_{1}, t^{\prime}\right) C^{p-2}\left(t_{1}, t^{\prime}\right) R\left(t^{\prime}, t_{2}\right)+\delta\left(t_{1}-t_{2}\right), \\
\left(m \partial_{t_{1}}^{2}+\gamma \partial_{t_{1}}+z\left(t_{1}\right)\right) C\left(t_{1}, t_{2}\right)-\frac{J^{2} p(p-1)}{2} \int_{0}^{t_{1}} \mathrm{~d} t^{\prime} R\left(t_{1}, t^{\prime}\right) C^{p-2}\left(t_{1}, t^{\prime}\right) C\left(t^{\prime}, t_{2}\right)+\gamma T R\left(t_{2}, t_{1}\right) \\
\quad\left|\frac{J^{2} p}{2} \int_{0}^{t_{2}} \mathrm{~d} t^{\prime} R\left(t_{2}, t^{\prime}\right) C^{p-1}\left(t_{1}, t^{\prime}\right)\right| \frac{J J_{0} p}{2 I^{\prime \prime}} C^{p-1}\left(t_{1}, 0\right) C\left(t_{2}, 0\right), \\
z\left(t_{1}\right)--\left.m \partial_{t_{1}}^{2} C\left(t_{1}, t_{2}\right)\right|_{t_{2} \rightarrow t_{1}^{-}}+\left.\gamma T R\left(t_{1}, t_{2}\right)\right|_{t_{2} \rightarrow t_{1}^{-}} \\
+\frac{J^{2} p^{2}}{2} \int_{0}^{t_{1}} \mathrm{~d} t^{\prime} R\left(t_{1}, t^{\prime}\right) C^{p-1}\left(t_{1}, t^{\prime}\right)+\frac{J J_{0} p}{2 T^{\prime}} C^{p}\left(t_{1}, 0\right) .
\end{gathered}
$$

One can check that these equations coincide with the ones in $[36,37\rfloor$ when inertia is neglected and $J-J_{0}$. 
In the rest of the paper we switch off the connection to the environment by setting $\gamma=0$. With inertia and no coupled bath, the cqual-time conditions are

$$
\begin{aligned}
C\left(t_{1}, t_{1}\right) & =1, \\
R\left(t_{1}, t_{1}\right) & =0, \\
\left.\partial_{t_{1}} C\left(t_{1}, t_{2}\right)\right|_{t_{2} \rightarrow t_{1}^{-}}=\left.\partial_{t_{1}} C\left(t_{1}, t_{2}\right)\right|_{t_{2} \rightarrow t_{1}^{-}} & =0, \\
\left.\partial_{t_{1}} R\left(t_{1}, t_{2}\right)\right|_{t_{2} \rightarrow t_{1}^{-}} & =\frac{1}{m}, \\
\left.R\left(t_{1}, t_{2}\right)\right|_{t_{2} \rightarrow t_{1}^{-}} & =0,
\end{aligned}
$$

for all times $t_{1}, t_{2}$ larger than $0^{+}$, when the dynamics start.

\subsection{The Lagrange multiplier}

We found convenient to numerically integrate the integro-differential equations that determine the time-evolution of the system to use an expression of the Lagrange multiplier that trades the second-time derivative of the correlation function into the total conserved energy after the quench. More precisely, we proceeded as explained below.

Firstly, we provide an expression for the kinetic energy density,

$$
e_{\mathrm{kin}}\left(l_{1}\right) \equiv \frac{E_{\mathrm{kin}}\left(t_{1}\right)}{N}=\frac{m}{2 N} \sum_{i}\left\langle\dot{s}_{i}^{2}\left(t_{1}\right)\right\rangle .
$$

Using the definition of $C\left(t_{1}, t_{2}\right)$, and the fact that, for sufficiently short time differences $\left(t_{1}-t_{2}\right)$ it is always possible to write $C\left(t_{1}, t_{2}\right)-1-a\left(t_{1}-t_{2}\right)^{2}+\mathcal{O}\left(\left(t_{1}-t_{2}\right)^{4}\right)$, with $a>0$, we find that

$$
e_{\mathrm{kin}}\left(t_{1}\right)=-\left.\frac{m}{2} \partial_{t_{1}}^{2} C\left(t_{1}, t_{2}\right)\right|_{i_{2} \rightarrow i_{1}^{-}} .
$$

On the other hand, the potential energy is linked to the kinetic energy and the Lagrange multiplier via a general equation proven as follows. Take the microscopic evolution of $s_{i}\left(t_{1}\right)$, multiply it by $s_{i}\left(t_{2}\right)$, and take the average over initial conditions:

$m\left\langle\ddot{s}_{i}\left(t_{1}\right) s_{i}\left(t_{2}\right)\right\rangle=-p \sum_{i_{2} \ldots i_{p}} J_{i i_{2} \ldots i_{p}}\left\langle s_{i}\left(t_{2}\right) s_{i_{2}}\left(t_{1}\right) \ldots s_{i_{p}}\left(t_{1}\right)\right\rangle-z\left(t_{1}\right)\left\langle s_{i}\left(t_{1}\right) s_{i}\left(t_{2}\right)\right\rangle$.

Summing now over $i$, normalising by $N$, and taking the limit $t_{2} \rightarrow t_{1}^{-}$,

$$
\frac{m}{N} \lim _{t_{2} \rightarrow t_{1}^{-}} \sum_{i}\left\langle\ddot{s}_{i}\left(t_{1}\right) s_{i}\left(t_{2}\right)\right\rangle=m \lim _{t_{2} \rightarrow t_{1}^{-}} \partial_{t_{1}^{2}} C\left(t_{1}, t_{2}\right)=-p e_{\text {pot }}\left(t_{1}\right)-z\left(t_{1}\right) .
$$

This implies that

$$
e_{\text {pot }}\left(t_{1}\right) \equiv \frac{E_{\text {polt }}\left(t_{1}\right)}{N}=\frac{-\left.m \partial_{t_{1}}^{2} C\left(t_{1}, t_{2}\right)\right|_{t_{2} \rightarrow t_{1}^{-}}-z\left(t_{1}\right)}{p} .
$$

The two contributions added together yield the total energy density of the system

$$
e\left(l_{1}\right)=e_{\text {kin }}\left(l_{1}\right)+e_{\text {pot }}\left(l_{1}\right) .
$$

conserved after the quench. 
Rearranging now the equation for $e\left(t_{1}\right)$, equation (58), with the help of equation (56), we obtain a new expression for the Lagrange multiplier

$$
z\left(t_{1}\right)=-\left.m\left(\frac{p}{2} \mid 1\right) \partial_{t_{1}}^{2} C\left(t_{1}, t_{2}\right)\right|_{\iota_{2} \rightarrow t_{1}^{-}}-p e\left(t_{1}\right) .
$$

Using now the original equation for $z\left(t_{1}\right)$, equation (52), we can eliminate the second time derivative to obtain

$z\left(l_{1}\right)=2 e\left(l_{1}\right)+\frac{J^{2} p(p+2)}{2} \int_{0}^{t_{1}} \mathrm{~d} \iota^{\prime} R\left(t_{1}, t^{\prime}\right) C^{p-1}\left(t_{1}, t^{\prime}\right)+\frac{J J_{0}(p+2)}{2 T^{\prime}} C^{p}\left(t_{1}, 0\right)$.

It scems that we have simply traded $z\left(t_{1}\right)$ by $e\left(t_{1}\right)$. However, for an isolated system $e\left(l_{1}\right)=e_{f}$, a constant. Then, the last expression allows a straightforward numerical solution of the evolution equations for the isolated system since it does not involve the second time derivative of the correlation function. In practice, in the numerical algorithm we fix the total energy $e_{f}$ and we then integrate the set of coupled integrodifferential equations with a standard Runge-Kutta method. We only have to define which is the total energy density of the system, the subject of the next two sections.

Before moving on, it is important to note that the Lagrange multiplier in equation (59) fixes the total energy and the spherical constraint only on average over the initial conditions ensemble. This means that if we consider the trajectory that satisfies the Newton equation (7) with an initial configuration specified by arbitrary values of $\left\{s_{i}(0), \dot{s}_{i}(0)\right\}$, with $z(t)$ given by equation (59), such trajectory will not, in general, satisfy the spherical constraint nor have a constant energy density.

\subsection{Energy change}

We now determine the energy changes induced by a quench in the disorder exchanges and a quench in the mass of the particle. As these two act separately on the potential and kinetic contributions to the total energy, the total energy change is the sum of the two variations.

3.3.1. The energy change after a potential energy quench. Let us investigate what is the change in energy density induced by the change in potential energy $J_{i_{1} \ldots i_{p}}^{0} \mapsto J_{i_{1} \ldots i_{p}}$, whilc keeping the mass constant $m_{0}=m$.

The energy density just before the quench is the energy density of a canonical equilibrium paramagnetic state at temperature $T^{\prime}$ and it is given by

$$
c_{i}=c\left(0^{-}\right)=\frac{T^{\prime}}{2}-\frac{J_{0}^{2}}{2 T^{\prime}} .
$$

The first term is the equipartition of the kinetic energy and the second one is the potential energy of the paramagnet in equilibrium. Note that this is still true if we choose $T_{s}^{0}<T^{\prime}<T_{d}^{0}$, since, although metastable states still dominate the energy landscape in that range of temperatures, the thermodynamics of the equilibrium states is indistinguishable from the one of the paramagnet (see section 2.2.2). 
Non equilibrium dynamics of isolated disordered systems: the classical Hamiltonian p-spin model

The energy density at time $t_{1}-0^{+}$right after the instantaneous quench is

$\epsilon\left(0^{+}\right)-e_{\text {kin }}\left(0^{+}\right)+\epsilon_{\text {pot }}\left(0^{+}\right)--\left.\frac{m}{2} \partial_{t_{1}}^{2} C\left(t_{1}, t_{2}\right)\right|_{t_{2}-t_{1}=0^{+}}+\frac{-\left.m \partial_{t_{1}}^{2} C\left(t_{1}, t_{2}\right)\right|_{t_{2} \rightarrow t_{1}^{-}=0^{+}}-z\left(0^{+}\right)}{p}$

Using the fact that with no mass change the kinetic energy does not vary between $t-0^{-}$and $t-0^{+}$

$$
-\left.\frac{m}{2} \partial_{t_{1}}^{2} C\left(t_{1}, t_{2}\right)\right|_{t_{2} \rightarrow t_{1}^{-}=0^{+}}=\frac{T^{\prime \prime}}{2}
$$

as confirmed numerically in section 5 , and the value of the Lagrange multiplier evaluated from equation (52) at $t=0^{+}$is

$$
z\left(0^{+}\right)=T^{\prime}+\frac{J J_{0 p}}{2 T^{\prime \prime}}
$$

we find

$$
e_{f} \equiv e\left(0^{1}\right)=\frac{T^{i}}{2}-\frac{J J_{0}}{2 T^{\prime}} .
$$

Equations (60) and (61) imply that the amount of energy injected during the instantaneous quench $J_{i_{1} \ldots i_{p}}^{0} \mapsto J_{i_{1} \ldots i_{p}}$ is

$$
\Delta e=e_{f}-e_{i}=\Delta e_{\mathrm{pot}}=\frac{. J_{0}\left(. J_{0}-J\right)}{2 T^{\prime}} .
$$

Therefore, $\Delta e>0$ if $J_{0}>J$ and $\Delta e<0$ if $J_{0}<J$.

3.3.2. The energy change after a quench in the mass. If we apply a quench in the mass, $m_{0} \mapsto m$, while leaving the random exchanges fixed, the energy balance is modified.

Imagine that we initialise the system in a paramagnetic or TAP state such that $e_{\text {pot }}\left(0^{-}\right)=-J^{2} /\left(2 T^{\prime}\right)$. If we change the mass according to $m_{0} \rightarrow m$, the potential energy does not change during the instantaneous quench. Instead, the kinetic energy does. Right before the quench the kinetic energy density is

$$
\epsilon_{\mathbf{k i n}}\left(0^{-}\right)-\frac{m_{0}}{2}\left(\dot{s}_{i}\left(0^{-}\right)\right)^{2}-\frac{T^{\prime}}{2} .
$$

while right after the quench the velocities have not changed but the mass of the particle has. Therefore,

$$
e_{\text {kin }}\left(0^{+}\right)=\frac{m}{2}\left(\dot{s}_{i}\left(0^{+}\right)\right)^{2}=\frac{m}{2}\left(\dot{s}_{i}\left(0^{-}\right)\right)^{2}=\frac{m}{m_{0}} \frac{T^{\prime}}{2} .
$$

The total energy after the quench is

$$
e_{f}=\frac{m}{m_{0}} \frac{T^{\prime}}{2}-\frac{J^{2}}{2 T^{\prime}}
$$

and the energy input by the quench reads

$$
\Delta e=e_{f}-e_{i}=\Delta e_{\text {kin }}=\left(\frac{m}{m_{0}}-1\right) \frac{T^{\prime}}{2} .
$$


Adding together the energy variation due to the the potential and mass quenches, the total cnergy change becomes

$$
\Delta e_{\mathrm{tot}}=\left(\frac{m}{m_{0}}-1\right) \frac{T^{\prime}}{2}+\frac{J_{0}^{2}}{2 T^{\prime}}\left(1-\frac{J}{J_{0}}\right) .
$$

\section{Asymptotic analysis}

Depending on the pre and post quench parameters the system reaches different asymptotic dynamics. In some cases, the system reaches a stationary regime but, for parameters carefully tuned, a final state with non stationary ageing behaviour can also be attained.

Before entering into the deduction of the asymptotic equations, we present the general reasoning that we use to find them.

We will first analyse in section 4.1 the cases in which a stationary regime is reached after the quench. This means that

- Weassumetime-translationalinvariance(TTI) $C\left(t_{1}, t_{2}\right) \mapsto C_{\text {st }}(\tau), R\left(t_{1}, t_{2}\right) \mapsto R_{\text {st }}(\tau)$ with $\tau=\iota_{1}-\iota_{2}$ and

- the fluctuation-dissipation theorem $R_{\mathrm{st}}(\tau)--1 / T_{f} d_{\tau} C_{\mathrm{st}}(\tau)$ for $\tau \geqslant 0$.

- We define the asymptotic limits of the correlation with the initial configuration $\lim _{t_{1} \rightarrow \infty} C\left(\iota_{1}, 0\right) \mapsto q_{0}$,

- and between two dynamic configurations $\lim _{\tau \rightarrow \infty} C_{\text {st }}(\tau)=q$.

- We assume that the kinetic energy density approaches $\lim _{t_{1} \rightarrow \infty} e_{\text {kin }}\left(t_{1}\right)-T_{f} / 2$ after the quench.

Clearly, all these assumptions can and have been verified numerically. The temperature of the final state, $T_{f}$, has to be calculated and the parameters $q_{0}$ and $q$ as well.

We anticipate that the parameters $q_{0}$ and $q$ will find two interesting interpretations in the cases in which the system is initially in a non-trivial TAP state. The value qo represents the overlap between a typical configuration of the TAP state of the pre-quench potential in which the system was prepared initially, and a typical configuration of the TAP state into which the original state has evolved in the post quench potential, if it still exists. Instead, $q$ is the self-overlap within the TAP state of the post-quench potential. This description will become clear after presenting the analytical and numerical results.

We will then analyse, in section 4.2 , the cases in which the system, starting from equilibrium in a disordered paramagnetic state at high temperature is set, after the quench, on the threshold level and the stationarity assumption fails. This is in agreement with what was expected from the properties of the states on the threshold, that are flat, and on which ageing properties were obtained after quenches from random initial conditions in the dissipative setting [17,31]. For these cases we need to modify the assumptions above and allow for a two-time scale dependence of the correlation and linear response functions that take a form with a separation of time-scales, as in equations (26) and (27). This 
Ansatz is introduced in the dynamic equations for $C$ and $R$, equations (50)-(52), and the evolution in two two-time sectors is studied separately together with the requirement that the behaviour matches in the crossover region. The resulting equations are manipulated a bit, and equations for the parameters $q, T_{f}$ and $T_{\text {eff, }}$ are derived. We reckon that with this procedure we introduce three unknowns and we deduce five equations, one being the energy conservation. The other four equations are the equations for $C_{\mathrm{st}}, C_{\mathrm{ag}}, R_{\mathrm{st}}$ and $R_{\mathrm{ag}}$, but these are not all independent, since the FDT with $T_{f}$ for $\left(C_{\mathrm{st}}, R_{\mathrm{st}}\right)$ and $T_{\text {eff }}$ for $\left(C_{\mathrm{ag}}\right.$, $R_{\mathrm{ag}}$ ) reduce their number to two. There are then three unknowns and three equations.

\subsection{Stationary dynamics}

In this section we derive the set of equations that determine $q, q_{0}$ and $T_{f}$ as a function of the properties of the initial state, $T^{\prime}, m_{0}$ and $J_{0}$, and the ones after the quench, $m$ and $J$, assuming that a stationary state is reached.

The stationarity assumption implies

$\lim _{t_{1} \rightarrow \infty} z\left(t_{1}\right)-z_{f}, \quad \lim _{t_{2} \rightarrow \infty} C\left(t_{1}, t_{2}\right)-C_{\mathrm{st}}\left(t_{1}-t_{2}\right), \quad \lim _{t_{2} \rightarrow \infty} R\left(t_{1}, t_{2}\right)-R_{\mathrm{st}}\left(t_{1}-t_{2}\right)$,

where we took $t_{1} \geqslant t_{2}$. The large $\tau \equiv t_{1}-t_{2}$ limit can then be further considered to define

$$
q \equiv \lim _{\tau \rightarrow \infty} C_{\text {st }}(\tau)
$$

This asymptotic limit has to be distinguished from the one of the correlation between the initial condition and the dynamic configuration

$$
q_{0} \equiv \lim _{t_{1} \rightarrow \infty} C\left(t_{1}, 0^{+}\right) \text {. }
$$

The parameters $q$ and $q_{0}$ will take zero or non-vanishing values in different situations presented below. Accordingly, $C_{\text {st }}(0) \neq C(0,0)$ since in the former equation the $t_{2} \rightarrow \infty$ limit has been taken and in the latter equation $t_{2}=0^{+}$. In the following presentation we drop the superscript + from the initial time but the 0 of the absolute times should be understood as $0^{\prime}$.

If $R_{\mathrm{st}}$ and $C_{\mathrm{st}}$ satisfy FDT with respect to a temperature $T_{f}$, and we call $\tau=t_{1}-t_{2}$,

$$
R_{\mathrm{st}}(\tau)=-\frac{1}{T_{f}} d_{\tau} C_{\mathrm{st}}(\tau) \quad \text { and } \quad \chi_{\mathrm{st}}(\tau) \equiv \int_{0}^{\tau} \mathrm{d} \tau^{\prime} R_{\mathrm{st}}\left(\tau^{\prime}\right)=\frac{1}{T_{f}}\left[1-C_{\mathrm{st}}(\tau)\right] \text {, }
$$

where we used $C_{\mathrm{st}}(0)-1$. The second way of writing the FDT is the one that we will exploit in the numerical analysis to determine the final temperature $T_{f}$ from the plot of $\chi_{\text {st }}$ against $C_{\text {st }}$ constructed using the time-lag $\tau$ as a parameter.

In order to make the presentation of the analytic part easier we list here the steps followed in the derivation of the asymptotic equations under the stationary assumption:

- We take the asymptotic limit of the equation for $z\left(t_{1}\right)$ and write $z f \equiv \lim _{t_{1} \rightarrow \infty} z\left(l_{1}\right)$ as a function of the

$q_{0}$ and $q$ parameters and $T_{j}$. 
Non equilibrium dynamics of isolated disordered systems: the classical Hamiltonian p-spin model

- We write the conservation of the energy.

- We prove that the equation for $R_{\mathrm{st}}$ becomes the $\tau$-derivative of the $C_{\mathrm{st}}$ equation.

- Wo take the asymptotic limit of the equations for $C\left(t_{1}, 0\right)$ and $C_{\mathrm{st}}(\tau)$.

The conservation of the total energy and the two last equations derived constitute a set that fixes $T_{f}, q_{0}$ and $q$ knowing $T^{\prime}, J_{0}, J, m_{0}$ and $m$. We do not prove analytically that the asymptotic solution is reached by the dynamics, this would need the full solution of the equations of motion and a matching problem that remains out of reach analytically. In contrast, we do verify a posteriori for which set of parameters $\left(T^{\prime}, J_{0}, J, m_{0}, m\right)$ this occurs by solving numerically the full set of equations.

The steps followed in the case in which the system ages and stationarity is broken are rather similar but need some generalisation, see section 4.2 .

4.1.1. The asymptotic Lagrange multiplier and the total energy. Starting from cquation (59) and using FDT

$z\left(t_{1}\right)=2 e_{f}+\frac{J^{2} p(p+2)}{2} \int_{0}^{t_{1}} \mathrm{~d} t^{\prime} \frac{1}{T_{f}}\left(\partial_{t^{\prime}} C\left(t_{1}, t^{\prime}\right)\right) C^{p-1}\left(t_{1}, t^{\prime}\right)+\frac{J J_{0}(p+2)}{2 T^{\prime}} C^{p}\left(t_{1}, 0\right)$

the integral can be computed and an asymptotic expression for $z_{f}$ is obtained

$$
z_{f}=2 e_{f}+\frac{J^{2}(p+2)}{2 T_{f}}\left(1-q^{p}\right)+\frac{J J_{0}(p+2)}{2 T^{\prime}} q_{0}^{p} .
$$

Proceding similarly, the potential energy is given by

$$
e_{\text {pot }}^{f}=-\frac{J^{2}}{2 T_{f}}\left(1-q^{p}\right)-\frac{J J_{0}}{2 T^{\prime}} q_{0}^{p}
$$

that becomes the paramagnetic result $e_{\text {pot }}^{f}=-J^{2} /\left(2 T_{f}\right)$ for $q_{0}-q-0$. Morcover, if $J-J_{0}$ and $T_{s}^{\prime} \leqslant T^{\prime}=T_{f}^{\prime}$ (no potential energy quench), $q_{0}=q$ and $e_{\text {pot }}^{f}=-J^{2} /\left(2^{\prime} T_{f}^{\prime}\right)$, independently of $q$, as it should. Note that we need the contribution from the last term to get the correct no-quench limit. Besides, we assume that the asymptotic kinetic energy is determined by 'equipartition' at the final temperature

$$
e_{\mathrm{kin}}^{f}=\frac{T_{f}}{2} .
$$

Then, the asymptotic total energy reads

$$
e_{f}=e_{\mathrm{kin}}^{f}+e_{\mathrm{pot}}^{f}=\frac{T_{f}}{2}-\frac{J^{2}}{2 T_{f}}\left(1-q^{p}\right)-\frac{J J_{0}}{2 T^{\prime}} q_{0}^{p} .
$$

We argued that the encrgy right after a quench in the interactions and mass is $e_{f}=e\left(0^{+}\right)=m l^{\prime \prime} /\left(2 m_{0}\right)-J J_{0} /\left(2 I^{\prime \prime}\right)$. Compared to the asymptotic form derived in equation (75) the conservation of the total energy implies

$$
\frac{m J_{0}}{m_{0} J} \frac{T^{\prime}}{T_{0}}-\frac{J_{0}}{T^{\prime}}=\frac{T_{f}}{J}-\frac{J}{T_{f}}\left(1-q^{p}\right)-\frac{J_{0}}{T^{\prime}} q_{0}^{p} .
$$


We see here two adimensional parameters $T^{\prime} / J_{0}$ and $J m_{0} /\left(J_{0} m\right)$ that characterise the pre-quench conditions and the comparison between the pre and post quench parameters.

The cquation for $z_{f}$ in equation (72) can now be rewritten as

$$
z_{f}=T_{f}+\frac{J^{2} p}{2 T_{f}}\left(1-q^{p}\right)+\frac{J J_{0} p}{2 T^{\prime}} q_{0}^{p}
$$

after replacing the energy $e_{f}$ by its dependence on $T_{f}$. It takes now a form that is very similar to the one of the relaxation dynamics [17].

4.1.2. The asymptotic analysis of the correlation equation. The equation for $C$ can be treated in two regimes of times. In one case we take $\tau-t_{1}-t_{2}$ fixed and $t_{1}$ and $t_{2}$ tending to infinity. The equation for $C$ then reads

$$
\begin{aligned}
{\left[m d_{\tau^{2}}+z_{f}\right] C_{\mathrm{st}}(\tau) } & =\frac{J^{2} p}{2 T_{f}} \int_{0}^{l_{2} \rightarrow \infty} \mathrm{d} t^{\prime} \frac{\partial}{\partial t^{\prime}}\left(C_{\mathrm{st}}^{p-1}\left(t_{1}, t^{\prime}\right) C_{\mathrm{st}}\left(t_{2}, t^{\prime}\right)\right) \\
& +\frac{J^{2} p}{2 T_{f}} \int_{0}^{\tau} \mathrm{d} \tau^{\prime}\left(\partial_{\tau^{\prime}} C_{\mathrm{st}}^{p-1}\left(\tau-\tau^{\prime}\right)\right) C_{\mathrm{st}}\left(\tau^{\prime}\right)+\frac{J J_{0} p}{2 T^{\prime}} C^{p-1}\left(l_{1}, 0\right) C\left(\ell_{2}, 0\right) .
\end{aligned}
$$

When deriving this equation we assumed that the contribution to the integrals of any possible transient between the time 0 and a time $t_{\mathrm{tr}}$ after which the FDT establishes can be neglected. The lower limit 0 in the integral over $l^{\prime}$ is then to be interpreted as the initial time $t_{\mathrm{tr}}$ of this asymptotic regime, although we simply write 0 in these equations. In the second integral 0 is the minimal time-delay at which the functions $C_{\mathrm{st}}$ and $R_{\mathrm{st}}$ are measured.

We can treat in the same way the equation for $R$ and then compare the two. As already mentioned in the list that summarizes the steps to follow, if we assume the fluctuation dissipation relation, it is possible to prove that the equation for $R_{\text {st }}(\tau)$ reduces to the time-delay derivative of the equation for $C_{\mathrm{st}}(\tau)$ times $1 / T_{f}$. This is a quite straightforward calculation that we choose not to show here.

In the limit $t_{1} \geqslant t_{2} \rightarrow \infty$ we can replace $C\left(t_{1}, 0\right)$ and $C\left(t_{2}, 0\right)$ by $q_{0}$. We further take the limit $\tau \rightarrow \infty$ and drop the second time derivative assuming that the dynamics become slow at long time delays. The first integral is computed as it is written now. The second one is made more symmetric before approximating it, in such a way that the two extremes $(0$ and $\tau$ ) contribute in the same way. One has

$$
\begin{aligned}
z_{f} q= & \frac{J^{2} p}{2 T_{f}}\left(q^{p-1}-q^{p}\right) \\
& +\frac{J^{2} p}{2 T_{f}} \lim _{\tau \rightarrow \infty} \int_{0}^{\tau} \mathrm{d} \tau^{\prime}\left[\frac{1}{2}\left(d_{\tau^{\prime}} C^{p-1}\left(\tau-\tau^{\prime}\right)\right) C\left(\tau^{\prime}\right)+\frac{1}{2} d_{\tau^{\prime}}\left(C^{p-1}\left(\tau-\tau^{\prime}\right) C\left(\tau^{\prime}\right)\right)-\frac{1}{2} C^{p-1}\left(\tau-\tau^{\prime}\right) d_{\tau^{\prime}} C\left(\tau^{\prime}\right)\right] \\
& +\frac{J J_{0} p}{2 T^{\prime}} q_{0}^{p} \\
= & \frac{J^{2} p}{2 T_{f}}\left(q^{p-1}-q^{p}\right)+\frac{J^{2} p}{2 T_{f}} \frac{1}{2}\left[q\left(1-q^{p-1}\right)+\frac{1}{2}\left(q-q^{p-1}\right)-\frac{1}{2} q^{p-1}(q-1)\right]+\frac{J J_{0} p}{2 T^{\prime}} q_{0}^{p} .
\end{aligned}
$$

Finally,

$$
z_{f} q=\frac{J^{2} p}{2 T_{f}}\left(q^{p-1}-q^{p}\right)+\frac{J^{2} p}{2 T_{f}} q\left(1-q^{p-1}\right)+\frac{J J_{0} p}{2 T^{\prime}} q_{0}^{p} .
$$


Non equilibrium dynamics of isolated disordered systems: the classical Hamiltonian p-spin model

This equation admits the solution $q_{0}-q-0$ but it can also have, for certain values of the parameters, solutions with $q \neq 0$ and $q_{0} \neq 0$ being equal or different.

The other interesting limit is the one in which we set $t_{2}$ to be strictly 0 and we tend $t_{1}$ to infinity. The equation for $C$ becomes

$$
\left[m d_{t_{1}^{2}}+z_{f}\right] C\left(t_{1}, 0\right)=\frac{J^{2} p(p-1)}{2} \int_{0}^{t_{1}} \mathrm{~d} t^{\prime} R\left(t_{1}, t^{\prime}\right) C^{p-2}\left(t_{1}, t^{\prime}\right) C\left(t^{\prime}, 0\right)+\frac{J J_{0} p}{2 T^{\prime}} C^{p-1}\left(t_{1}, 0\right) C(0,0) .
$$

In the limit $t_{1} \rightarrow \infty$ we can replace $C\left(t_{1}, 0\right)$ by $q_{0}$ and use $C(0,0)-1$. We further drop the second time derivative, and use stationarity and FDT, to find

$$
z_{f} q_{0}-\frac{J^{2} p}{2 T_{f}} q_{0}\left(1-q^{p-1}\right)+\frac{J J_{0} p}{2 T^{\prime}} q_{0}^{p-1} .
$$

This equation admits the solution $q_{0}=0$ but it can also have, for certain values of the parameters, solutions with $q_{0} \neq 0$. As a check of consistency, we remark that for $J=J_{0}$ and $T^{\prime}-T_{f}$ the two remaining equations, equations (80) and (82), are compatible for $q-q_{0}$.

We can now write down two other equations that relate $q_{0}, q$ and $T_{f}$.

$$
\begin{gathered}
{\left[T_{f}+\frac{J^{2} p}{2 T_{f}}\left(1-q^{p}\right)+\frac{J J_{0} p}{2 T^{\prime}} q_{0}^{p}\right] q_{0}=\frac{J^{2} p}{2 T_{f}}\left(1-q^{p-1}\right) q_{0}+\frac{J J_{0} p}{2 T^{\prime}} q_{0}^{p-1},} \\
{\left[T_{f}+\frac{J^{2} p}{2 T_{f}}\left(1-q^{p}\right)+\frac{J J_{0} p}{2 T^{\prime}} q_{0}^{p}\right] q=\frac{J^{2} p}{2 T_{f}} q^{p-1}(1-q)+\frac{J^{2} p}{2 T_{f}} q\left(1-q^{p-1}\right)+\frac{J J_{0} p}{2 T^{\prime}} q_{0}^{p} .}
\end{gathered}
$$

4.1.3. The equations fixing the parameters $q$, $q_{0}$ and $T_{j}$. After some rearrangements, the three equations (76), (83) and (84) simplify to

$$
\begin{aligned}
& \frac{J_{0} m}{J m_{0}} \frac{T^{\prime}}{J_{0}}-\frac{T_{f}}{J}=-\frac{J}{T_{f}}\left(1-q^{p}\right)+\frac{J_{0}}{T^{\prime}}\left(1-q_{0}^{p}\right), \\
& q_{0} \frac{T_{f}}{J}=-\frac{p}{2} \frac{J}{T_{f}} q_{0} q^{p-1}(1-q)+\frac{p}{2} \frac{J_{0}}{T^{\prime}} q_{0}^{p-1}\left(1-q_{0}^{2}\right) . \\
& q \frac{T_{f}}{J}=\frac{p}{2} \frac{J}{T_{f}} q^{p-1}(1-q)^{2}+\frac{p}{2} \frac{J_{0}}{T^{\prime}} q_{0}^{p}(1-q) .
\end{aligned}
$$

One can use equations (85)-(87) to determine $q_{0}, q, T_{f}$ in situations in which a steady stiate is reached.

More simplifications are possible if one extracts $q^{p}(1-q) / q$ from the second equation and inserts it in the third one to obtain

$$
\frac{I_{f}}{J} \frac{I^{\prime \prime}}{J_{0}}=\frac{p}{2} q_{0}^{p-2}(1-q)
$$

a linear equation in $q$.

We can now check that for $J=J_{0}$ and $m=m_{0}$, the equation that expresses energy conservation is consistent with $T^{\prime}-T_{f}$ and $q=q_{0}$. Moreover, taking $q_{0}=q$, equations (87) and (88) become the same one, 
Non equilibrium dynamics of isolated disordered systems: the classical Hamiltonian p-spin model

$$
\frac{p}{2} q^{p-2}(1-q)=\left(\frac{T_{f}}{J}\right)^{2},
$$

that is the equation for $q$ in the TAP solutions that are mixed to yield the non-trivial paramagnet at $T_{s}^{0}<T^{\prime \prime}<T_{d}^{0}$, see equation (23). The dynamics remain confined in the initial TAP state where the system was prepared.

4.1.4. Quench dynamics, target paramagnetic state. Let us look for solutions with $q_{0}=q=0$ that correspond to a final paramagnetic state. Equations (86) and (87) are identical to zero and equation (85) implies

$$
\frac{J_{0} m}{J m_{0}} \frac{T^{\prime}}{J_{0}}-\frac{J_{0}}{T^{\prime}}=\frac{T_{f}}{J}-\frac{J}{T_{f}}
$$

that fixes $T_{f}$

$$
T_{f}^{(1,2)}=J \frac{J_{0}}{2 T^{\prime}}\left[\frac{J_{0} m}{J m_{0}}\left(\frac{T^{\prime}}{J_{0}}\right)^{2}-1 \pm \sqrt{\left[\frac{J_{0} m}{J m_{0}}\left(\frac{T^{\prime}}{J_{0}}\right)^{2}-1\right]^{2}+\left(\frac{2 T^{\prime}}{J_{0}}\right)^{2}}\right] .
$$

As the temperature cannot be negative, the plus sign is the relevant one here. This relation can be used to check whether the system has really attained thermal equilibrium by looking at the parametric plot of the integrated linear response, $\chi\left(t_{1}, t_{2}\right)=\int_{t_{1}}^{t_{2}} \mathrm{~d} t^{\prime} R\left(t_{1}, t^{\prime}\right)$, as a function of the correlation function, $C\left(t_{1}, t_{2}\right)$, and comparing minus the inverse slope with $T_{f}$. For a stationary system, the expected linear form is given in cquation (70).

The temperature in the asymptotic paramagnetic state, in units of $J$, is a function of $T^{\prime} / J_{0}$ and $J m_{0} /\left(J_{0} m\right)$. One can easily show from the analytic form above that $T_{f}=J\left(J_{0} m /\left(J m_{0}\right)\right)\left(T^{\prime} / J_{0}\right)$ at $m_{0} J /\left(m J_{0}\right) \rightarrow 0$. In the limit $J m_{0} /\left(J_{0} m\right) \rightarrow \infty$ the temperature approaches $T_{f} \rightarrow J /\left(2 T^{\prime}\right)\left(-J_{0} \mid \sqrt{J_{0}^{2} \mid 4 T^{\prime 2}}\right)$. If, moreover, $T^{\prime} / J_{0}$ also diverges, it becomes $T_{f}^{\prime} \rightarrow J$. Some special finite values are $T_{f}^{\prime}=J^{\prime} I^{\prime} / J_{0}$ for $m_{0} J /\left(m J_{0}\right)=1$, and $T_{f}-T^{\prime}$ if $m_{0}=m$ and $J_{0}=J$.

The curve $T_{f} / J$ as a function of the full control parameter $\left(J m_{0} /\left(J_{0} m\right)\right)$ is shown with dotted blue lines in the two panels in figure 2 that represent quenches from equilibrium at $T^{\prime}=0.7 J_{0}$ and $T^{\prime}=0.6 J_{0}$. The open circles correspond to dynamical runs that realise the paramagnetic asymptotic solution. We will discuss the region of parameters in which this is the asymptotic state in sections 5 and 6 .

The relation between the final total energy and the final temperature is very simple in the paramagnetic state

$$
T_{f}=e_{f}+\sqrt{e_{f}^{2}+J^{2}},
$$

and it coincides with the result of inverting equation (60), that is to say, the equilibrium paramagnetic encrgy as a function of temperature.

4.1.5. Dynamies within metastable states. When the temperature of the initial condition is such that $T_{s}^{0}<T^{\prime}<T_{d}^{0}$, the system is prepared in a TAP state. In this section we will show that whenever the asymptotic equations (85)-(87) have solutions with $q, q_{0} \neq 0$, the dynamics of the system are confined to the same TAP state that, 
after the change in the coupling strength operated at the quench, is only translated in the potential energy landscape and possibly rescaled in size, thanks to the fact that in the spherical p-spin model there is no birth, death or merging of states at intermediate temperatures.

The first remark is that, as already mentioned, the interaction quench changes the depth of the potential energy minima. If one minimum has encrgy $e_{\text {pot }}^{0}$ initially, its energy after the quench is given by

$$
e_{\text {pot }}=\frac{J}{J_{0}} e_{\text {pot }}^{0}
$$

Given that $T_{s}^{0}<T^{\prime}<T_{d}^{0}$, the initial state is described by equation (23) that we here rewrite making the dependence of the parameter $q$ on the initial temperature, $T^{\prime}$, and strength of the random potential, $J_{0}$,

$$
\frac{J_{0}^{2} p}{2 T^{\prime 2}} q\left[J_{0}, T^{\prime}\right]^{p-2}\left(1-q\left[J_{0}, T^{\prime}\right]\right)-1,
$$

explicit. Indeed, $q\left[J_{0}, T^{\prime}\right]$ is the equilibrium value of the self overlap at the initial temperature ' $T$ '. This equation can be written in a slightly different way that will be useful later

$$
q\left[J_{0}, T^{\prime}\right]-\left[\frac{J_{0}^{2} p}{2}\left(1-q\left[J_{0}, T^{\prime}\right]\right)\right]^{-\frac{1}{p-2}}\left(T^{\prime}\right)^{\frac{2}{p-2}}=0 .
$$

We call $e^{0}$, the bare potential energy of the TAP state that dominates the partition function at the initial temperature $T^{\prime}$. In such case, from equation (19), qT also fullfills

$$
(p-1)\left(1-q\left[J_{0}, T^{\prime}\right]\right) q\left[J_{0}, T^{\prime}\right]^{(p-2) / 2}=\frac{T^{\prime}}{J_{0}^{2}}\left[-e_{T^{\prime}}^{0}-\sqrt{\left(e_{T^{\prime}}^{0}\right)^{2}-e_{c}^{0}}\right],
$$

where $e_{c}^{0}--J_{0} \sqrt{2(p-1) / p}$. According to equation (93), after the quench, the energy of this TAP state is given by $e_{T^{\prime}}=\left(J / J_{0}\right) e_{T^{\prime}}^{0}$.

Let us call $q\left[J, T_{f}\right]$ the self overlap in the final TAP state at temperature $T_{f}$. Also from equation (19), and since the energy of the TAP state is rescaled, it is clear that $q\left[J, T_{f}\right]$ satisfies

$$
(p-1)\left(1-q\left[J, T_{f}\right]\right) q\left[J, T_{f}\right]^{(p-2) / 2}=\frac{T_{f}}{J^{2}}\left[-e_{T^{\prime}}-\sqrt{e_{T^{\prime}}^{2}-e_{c}}\right],
$$

where $e_{c}=-J \sqrt{2(p-1) / p}$. Recalling that $e_{T^{\prime}}-\left(J / J_{0}\right) e_{T^{\prime}}^{0}$, we can write

$$
\left.(p-1)\left(1-q\left|J . T_{f}\right|\right) q \mid J, T_{f}\right]^{(p-2) / 2}-\frac{T_{f}^{\prime}}{J J_{0}}\left[-e_{T^{\prime}}^{0}-\sqrt{\left(e_{T^{\prime}}^{0}\right)^{2}-e_{c}^{0}}\right] .
$$

Then, from equations (94), (96) and (98) we obtain a relation between $q\left[J_{0}, T^{\prime}\right]$ and $q\left[J, T_{f}\right]$

$$
q\left[J_{0}, T^{\prime}\right]=1-\frac{J^{2} p}{2 T_{f}^{2}}\left(1-q\left[J, T_{f}\right]\right)^{2} q\left[J, T_{f}\right]^{p-2}
$$


Using now the results for the asymptotic analysis of the dynamical equation, more precisely, cquations (87) and (88), the equation for $q-\lim _{\tau \rightarrow \infty} C(\tau)$ can be written as

$$
1=\frac{J^{2} p}{2 T_{f}^{2}} q^{p-2}(1-q)^{2}+\left[\frac{J J_{0} p}{2 T^{\prime} T_{f}}\right]^{-\frac{2}{p-2}} q^{-1}(1-q)^{-\frac{2}{p-2}} .
$$

Defining

$$
U \equiv 1-\frac{J^{2} p}{2 T_{f}^{2}} q^{p-2}(1-q)^{2},
$$

we can write equation (100) as

$$
U-\left[\frac{J_{0}^{2} p}{2}(1-U)\right]^{-\frac{1}{p-2}}\left(T^{\prime}\right)^{\frac{2}{p-2}}=0 .
$$

Comparing this last equation with equation (95) we conclude that

$$
U=q\left|\cdot J_{0}, T^{\prime}\right|
$$

which, using equation (99), implies

$$
q=q\left[J, T_{f}\right] .
$$

This shows that the system remains trapped in the same metastable state during all the evolution. Of course, if the quench takes the system to parameters (final temperature $T_{f}$ ) such that this state does no longer exist, the system escapes it into the proper paramagnetic state.

In figure 2(b) we draw, with blue dotted lines, the $I m_{0} /\left(J_{0} m\right)$ dependence of $T_{f}$ for the asymptotic TAP states and the open squares show the numerical solution of the full equations for two choices of $J m_{0} /\left(J_{0} m\right)$ that realise this asymptotic state.

\subsection{Non stationary dynamics and ageing}

Let us now explain how the ageing equations are studied. In the aging regime we expect the correlation with the initial configuration to decay to zero

$$
\lim _{t_{1} \rightarrow \infty} C\left(t_{1}, 0\right)=0 \text {. }
$$

The dynamic equations (50)-(52) therefore lose the terms that depend on the initial conditions. The only formal difference with the equations for the dissipative case [17, $23]$ is that the friction term (first time derivative) is now replaced by the inertial term (second-time derivative) and that the temperature is not fixed a priori.

4.2.1. The parameters $q, T_{f}$, and $T_{\text {eff. }}$ Following the explanation in [17], explained in more detail in [41], the study of the $C$ and $R$ yields the equation that fixes plateau parameter $q$ to be the one on the threshold, equation (33). In the stationary regime the temperature is given by the parameter $T_{f}$ in the FDT linking $C_{\text {st }}$ and $R_{\text {st }}$, that is not fixed yet. Moreover, the combination of the $C$ and $R$ equations in the stationary and aging regime yields the equation that fixes the effective temperature in the aging

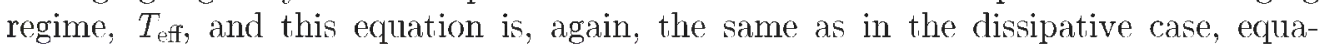
tion (34). We have 


$$
\begin{aligned}
& \frac{T_{f}^{2}}{J^{2}}=\frac{p(p-1)}{2} q^{p-2}(1-q)^{2}, \\
& \frac{T_{f}}{T_{\text {eff }}}=\frac{(p-2)(1-q)}{q} .
\end{aligned}
$$

It is not necessary to fix the value of the Lagrange multiplier to derive these two equations. We still need to find, though, which is the value of $T_{f}$ selected by the closed system.

The selection of $T_{f}$ is done by the energy conservation. The asymptotic energy is the sum of the kinetic contribution, $T_{f} / 2$, and the potential one that reads

$$
e_{\text {pot }}^{f}--\frac{J^{2}}{2}\left[\frac{1}{T_{f}}\left(1-q^{p}\right)+\frac{1}{T_{\text {eff }}} q^{p}\right] .
$$

Therefore

$$
\frac{J_{0} m}{J m_{0}} \frac{T^{\prime}}{J_{0}}-\frac{J_{0}}{T^{\prime}}=\frac{T_{f}}{J}-\left[\frac{J}{T_{f}}\left(1-q^{p}\right)+\frac{J}{T_{\mathrm{eff}}} q^{p}\right] .
$$

We now have three equations for the three unknowns $q, T_{f}$ and $T_{\text {eff }}$ These equations can be simplified and recast in a more convenient manner by replacing the q-dependence of $T_{j} / T_{\text {eff }}$ from equation (107) in equation (109), that is now a quadratic equation on $T_{f} / J$. Solving for $T_{f} / J$ and replacing the result in equation (106), after a straightforward calculation, we obtain

$\left(\frac{J_{0} m}{J m_{0}} \frac{T^{\prime}}{J_{0}}-\frac{J_{0}}{T^{\prime}}\right)^{2}=\frac{2 q^{2-p}}{p(p-1)(1-q)^{2}}\left[\frac{p(p-1)}{2} q^{p-2}(1-q)^{2}-\left(1-q^{p}\right)-(p-2)(1-q) q^{p-1}\right]^{2}$.

Equation (110) determines $q$ given the initial temperature $T^{\prime}$, the pre and post quench variance of the random interactions parametrized by $J_{0}$ and $J$ and the pre and post quench masses $m_{0}$ and $m$. These parameters appear in the combinations $T^{\prime} / J_{0}$ and $J m_{0} /\left(J_{0} m\right)$. Once $q$ is found, equations (106) and (107) yield $T_{f} / J$ and $T_{\text {eff }} / J$, respectively.

The solutions to equation (110) can be understood graphically. The rhs is a function of $q$ with positive curvature and a single minimum at $q-(p-2) / p$, the overlap at the spinodal, in the interval $q \in|0,1|$. The equation has two solutions, the one with larger value being the relevant one. When the control parameter $m_{0} J /\left(m J_{0}\right)$ is decreased, the equation ceases to have solution at

$$
\left(\frac{m_{0} J}{m J_{0}}\right)_{\min }=\frac{T^{\prime}}{J_{0}} \frac{1}{\frac{J_{0}}{T^{\prime}}+\sqrt{\text { rhs equation }(110)\left(\frac{p-2}{p}\right)}} .
$$

when the lhs touches the minimum of the rhs This value is $\approx 1.295$ for $T^{\prime} / J_{0}-0.7$ and $\approx 0.77$ for $T^{\prime} / J_{0}=0.6$, and $p=3$ (see the ending points of the ageing $T_{f} / J$ and $T_{\text {eff }} / J$ curves in figure 2 , and the discussion of the phase diagram in section 6 ).

In the ageing solutions, as long as $T_{f}$ (the temperature of the fast degrees of freedom) is lower than $T_{d}$, the effective temperature is larger than $T_{f}$. When $T_{f}$ goes beyond $T_{d}$, its relation with the effective temperature is inverted, and it becomes larger than $T_{\text {eff: }}$ 
Non equilibrium dynamics of isolated disordered systems: the classical Hamiltonian p-spin model

(a)

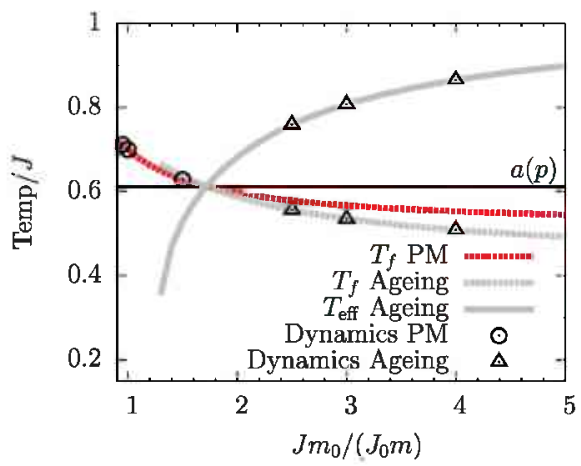

(b)

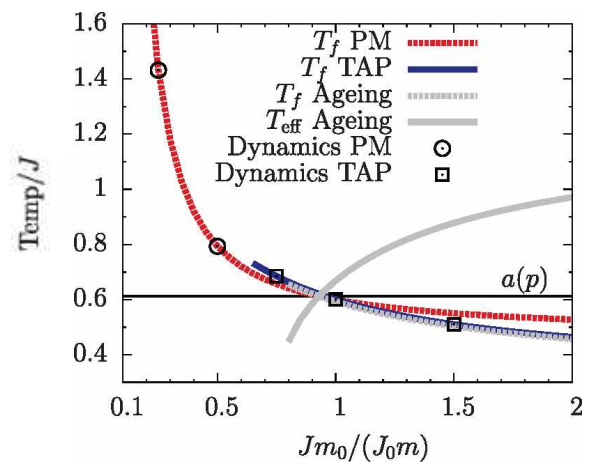

Figure 2. Characteristic temperatures in units of $J$ after quenches from (a) an initial paramagnetic state with $T^{\prime}=0.7 J_{0}>T_{d}^{0}$ and (b) an initial TAP state with $T^{\prime}=0.6 . J_{0}<T_{d}^{0}$. The lines represent the asymptotic solutions as indicated in the keys. The data points are the results of the numerical solution of the full set of equations. For each set of pre and post quench parameters one and only asymptotic state is realised. (a) ' $T^{\prime \prime}=0.7 J_{0}>T_{d}^{0}$. (b) $T^{\prime \prime}=0.6 J_{0}<T_{d}^{0}$.

$$
\begin{array}{lll}
T_{\text {eff }}>T_{f} & \text { for } & T_{d}>T_{f} \\
T_{\text {eff }}<T_{f} & \text { for } & T_{d}<T_{f} .
\end{array}
$$

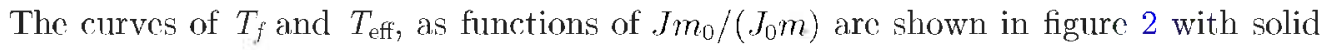
grey and dotted grey lines, respectively. The open triangles indicate the actual solution of the full equations found numerically. The temperature inversion, $T_{f}>T_{\text {off }}$ predicted by the asymptotic ageing equations for $T_{f}>T_{d}$ is not realised asymptotically by the full equations but, as we will show in section 5.2.3, it appears in a transient regime.

\subsection{Summary of asymptotic solutions}

As a summary of the different asymptotic solutions, we show in figure 2 the $m_{0} J /\left(m J_{0}\right)$-dependence of the dynamic critical temperature, $T_{d}$ from equation (37), the final temperature $T_{f}$ of the PM $\left(q-q_{0}-0\right)$ and TAP $\left(q, q_{0} \neq 0\right)$ branches of the solutions to equations (85)-(87) (stationary Ansatze), and the final temperature, $T_{F}$, and effective temperature, $T_{\text {eff }}$, derived from the solution to the set of equations (106)-(110) (ageing Ansatze). We note that the ageing temperatures $T_{f}$ and $T_{\text {eff }}$ coincide with $T_{d}$ at a single value of the control parameter $J m_{0} /\left(J_{0} m\right)$. We also show with different points the results from the full solution of the evolution equations, indicating which is the asymptotic solution realised by the dynamics in each range of parameters.

In panel figure 2(a), the initial state is paramagnetic $T^{\prime}=0.7 J_{0}>T_{d}^{0}$, and the dynamics choose the PM solution (red dotted line and open triangles) for energy injection or for small energy extraction, while for sufficiently large energy extraction the asymptotic dynamics show ageing, characterised by two temperatures (grey lines).

In panel figure 2(b), $T^{\prime}=0.6 . J_{0}<T_{d}^{0}$ and the initial configurations are drawn within a TAP state. The dynamics choose the PM solution (red dotted) for large energy 
Non equilibrium dynamics of isolated disordered systems: the classical Hamiltonian p-spin model

injection, while the system remains in a TAP state (blue lines and open triangles) for small energy injection or for energy extraction. The ageing solution (grey lines) are not realised by the dynamics.

The exact boundaries between the different kinds of solutions selected by the full equations will be derived analytically in section 6 . In general, transients appear in the parameter regions where the system changes its asymptotic behavior.

\section{Numerical results}

In the numerical solution of the full set of equations we fix $J_{0}-m_{0}-m-1$. This means that the initial energy landscape is fixed. In particular, we know the values of the initial critical temperatures $T_{d}^{0}-0.612$ and $T_{s}^{0}-0.586$. We shall then vary the initial temperature $T^{\prime \prime}$ and the coupling $J$ of the Hamiltonian that drives the time evolution.

For later reference and recalling the discussion in section 3, the critical temperatures corresponding to the equilibrium landscape with the final coupling $J$ can be calculated by noticing that the critical temperatures are proportional to the coupling. Then

$$
\frac{T_{s}}{T_{s}^{0}}-\frac{J}{J_{0}} \quad \Rightarrow \quad T_{\varepsilon}-\frac{J}{J_{0}} T_{s}^{0} \quad \text { and } \quad T_{d}-\frac{J}{J_{0}} T_{d}^{0} .
$$

After some general considerations about the numerical algorithm we analyse some specific processes to illustrate the analytical results of the previous section and put them to the test. We will consider energy injection and energy extraction processes sketched in figures 10 and 11, respectively. The full numerical solution to the equations allows to prove which among the asymptotic solutions are realised, when several co-exist.

\subsection{Equilibrium dynamics}

We first checked that for $J=J_{0}$ and $m=m_{0}$, that is to say $\Delta e=0$, the system has a stationary evolution for all equilibrium initial conditions.

We studied the no energy change case with two purposes. One is to check consistency of our numerical algorithm. The other is to investigate the effect of the discretisation step $\delta$ on the results obtained from the numerical integration of the equations. We found that the algorithm does conserve energy and that a step $\delta-0.0025$ was sufficient to assure numerical convergence of our results.

We used two typical cases as initial states, a paramagnetic configuration and a metastable TAP state. Figures 3 and 4 show three plots, with the dynamics of the correlation function (a), the fluctuation dissipation parametric plot (b) and the two contributions to the energy and the total energy (c), starting from equilibrium at $T^{\prime}=0.8$ and $T^{\prime}=0.6$, respectively. In both cases the system is paramagnetic initially, though at $T^{\prime}-0.8$ it is a proper paramagnet while at $T^{\prime}-0.6$ it is a paramagnetlooking state made of a mixture of non-trivial metastable states, see section 2 .

Let us first focus on figure 3 . From figure $3(\mathrm{a})$ we observe that the correlation with the initial condition, $C\left(t_{1}, 0\right)$ (thin red line) and the ones between two different times 
Non equilibrium dynamics of isolated disordered systems: the classical Hamiltonian p-spin model
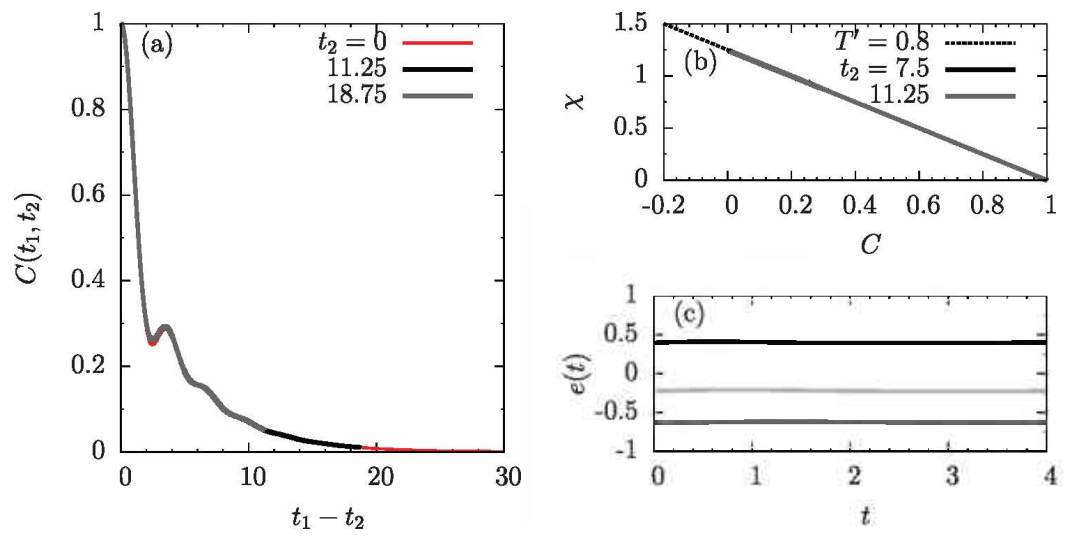

Figure 3. Constant energy dynanics, $\Delta c=0\left(J=J_{0}\right.$ and $\left.m=m_{0}\right) \cdot T^{\prime}=0.8>T_{d}^{0}$ in the paranagnetic phase. (a) Dynamics of the correlation function for various choices of the waiting time given in the key. The stationary relaxation to zero is clear. (b) Linear-response versus correlation parametric plot for two values of the waiting time $t_{2}$ indicated in the plot. The dashed line shows the FDT with the initial temperature. (c) Energy time-dependence. The data correspond to $e_{\text {kin }}$ (above), $e_{f}$ (middle), and $e_{\text {pot }}$ (below). The numerically evaluated values of the potential and kinetic energies and temperature agree with the ones derived analytically within numerical accuracy.

$C\left(t_{1}, t_{2}\right)$ (grey lines) lines are identical, apart from a small deviation at short timedelays, around the first oscillation. The two-time correlation function is invariant under time-translations, that is to say, it is a function of $t_{1}-t_{2}$ only. All the correlation functions relax to zero, $q_{0}=q=0$. The Lagrange multiplier (not shown) and the potential and kinetic energies quickly approach their final values and these agree with the ones predicted analytically, as secn in figure 3(c). The fluctuation-dissipation relation is satisfied with the temperature of the initial condition, that is the same as the one of the final state, see figure 3(b). All these results are compatible with equilibrium in the paramagnetic phase.

In figure 4 we show results for $T^{\prime \prime}=0.6$. The Lagrange multiplier (not shown) and energy densities approach constants (see figure $4(c)$ ), and stationarity is satisfied as well as the FDT with the initial temperature, as can be seen from figure 4(b). The main difference with the case $T^{\prime}=0.8$ is that the correlation functions, both with the initial condition and with the configuration at a waiting-time $t_{2}$, relax to a nonvanishing value (see figure 4(a)). Within numerical accuracy we observe $q_{0}=q \simeq 0.6$ and this value as well as the asymptotic potential and kinetic energies are consistent with the ones stemming from the analysis in section 4.1.2. One can use equation (89), that coincides with equation (23) and fixes the $q$ values of the non-trivial TAP states that correspond to equilibrium in the interval $\left[T_{s}^{0}, T_{d}^{0}\right][27]$, and check that the solution for $T_{f}-0.6$ is $q-0.6$, the value obtained with the numerical solution of the full dynamic equations. As regards the energy values, the kinetic energy should be $e_{\mathrm{kin}}^{f}=T_{f} / 2=0.3$ that is obtained numerically. The potential energy is expected to be 
Non equilibrium dynamics of isolated disordered systems: the classical Hamiltonian p-spin model
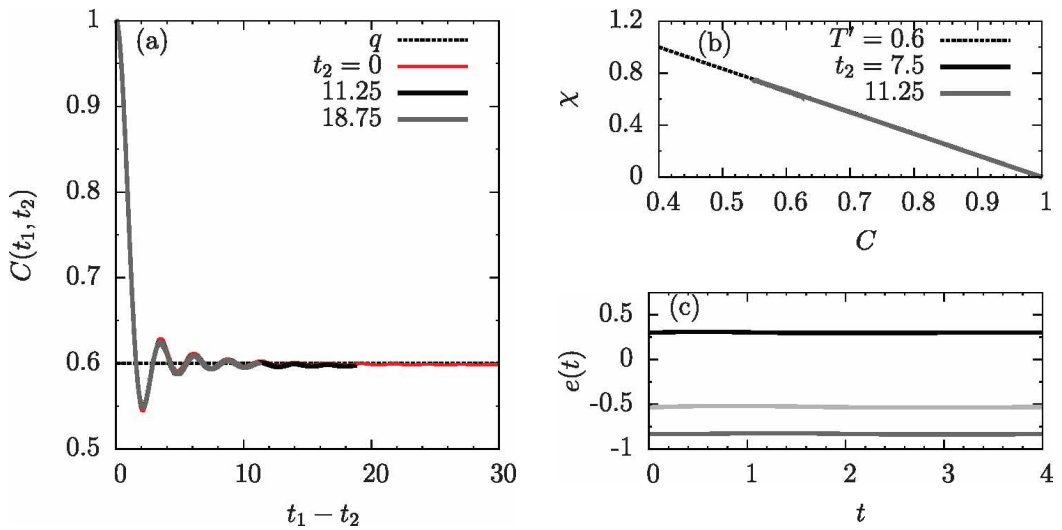

Figure 4. Constant energy dynamics in the temperature region $T_{s}^{0}<T^{\prime}=0.6<T_{d}^{0}$. As $J-J_{0}$ and $m-m_{0}$ there is no quench and $\Delta e-0$. (a) Stationary dynamics of the two-time correlation function. The asymptotic limit is $q \neq 0$ since $T^{\prime}<T_{d}^{0}$. (b) Linear-response versus correlation parametric plot. The dashed line shows the FDT with the initial temperature. (c) Energy time-dependence. The data correspond to $\epsilon_{\text {kim }}$ (above), $\epsilon_{f}$ (middle), and $\epsilon_{\mathrm{pot}}$ (below). All the numerically evaluated values of the parameters $q, T_{f}-T^{\prime}, \epsilon_{\mathrm{pot}}^{f}$, and $e_{\text {kin }}^{f}$ agree (within numerical accuracy) with the ones derived analytically. See the text for more details.

$e_{\text {pot }}^{f}--J^{2} /\left(2 T_{f}\right)--0.83$ which is also correct numerically. These values are added to $e_{f}=T_{f} / 2-J^{2} /\left(2 T_{f}\right)=-0.53$, as they should.

\subsection{Energy injection}

In this section we explore the dynamics after energy injection $\left(J<J_{0}\right.$ and $\left.m=m_{0}\right)$ and we compare our results with those obtained analytically in the previous section.

The injection of energy over an initial state with temperature $T^{\prime}>T_{d}^{0}$ trivially evolves into a paramagnetic state at a temperature $T_{f}>T^{\prime}$. The temperature can be calculated from the conservation of energy and the fact that for the PM phase $e_{\text {pot }}^{f}=-J^{2} /\left(2 T_{f}\right)$. It is given by equation (91). We have checked that the numerical solution complies with these claims (not shown here). Therefore, we shall focus on the more interesting cases with initial temperatures such that $I_{s}^{0}<T^{\prime \prime}<1_{d}^{0}$. Figure 10 summarises the results of the concrete numerical quenches with energy injection that we display.

5.2.1. $T_{s}^{0}<T^{\prime}<T_{d}^{0}$. from TAP to $P M$. In figure 5 we show results for $T_{s}^{0}<T^{\prime}=0.6<T_{d}^{0}$, $m-m_{0}$ and $J=0.25$. The system is initialised in a TAP state that corresponds to equilibrium between $T_{s}^{0}$ and $T_{d}^{0}$, see section 2.2.2. This quench injects a large amount of energy in the system $\Delta e,-0.625$. The post-quench critical temperatures are $T_{d}=0.153$ and $T_{s}=0.146$. The self correlations shown in figure 5(a) rapidly decay to zero for all reference times, either when they correspond to an initial $t_{2}-0^{+}$or to a waiting-time $t_{2}>0$. Therefore $q_{0}-q-0$. These facts indicate that the system behaves as in the paramagnetic state after the quench. The final temperature $T_{f}=0.358$ predicted by 
the asymptotic analysis, equation (91), is in very good agreement with the numerical result extracted from the parametric $\chi(C)$ plot in figure 5(b). Considering the results from section 4.1 .5 for quenches starting from a TAP state it is important to know the temperature $T_{\mathrm{TAP}}^{\max }$ at which the initial TAP state ceases to exist, i.e. the position of the spinodal line in the post quench energy landscape (see figure 1). For $T^{\prime}=0.6$ and $J=0.25, T_{\mathrm{TAP}}^{\max }=0.186339$. Note that $T_{f}>T_{\mathrm{TAP}}^{\max }$ which is consistent with the system reaching a paramagnetic state with $q=q_{0}=0$, although it was initialised in a nonergodic initial state, since for that final temperature the TAP state no longer exists. From the energetic dynamics figure $5(\mathrm{c})$ we observe $e_{\text {kin }}^{f}=T_{f}^{\prime} / 2$ and $e_{\text {pot }}^{f}=-J^{2} /\left(2^{\prime} T_{f}^{\prime}\right)$, both results consistent with equilibration in a paramagnctic final state. At very short times, $t_{1} \rightarrow 0^{+}$, the encrgies are the ones right after the quench, $e_{\text {kin }}\left(0^{+}\right)-T^{\prime} / 2$ and $e_{\text {pot }}\left(0^{+}\right)=-J J_{0} /\left(2 l^{\prime \prime}\right)$. It is only after a short transient, $t_{\mathrm{tr}} \simeq 1$, that the energy densities converge to their final values.

5.2.2. $T_{s}^{0}<T^{\prime}<T_{d}^{0}$ : from $T A P$ to $T A P$. In figure 6 we show results for $T_{s}^{0}<T^{\prime}=0.6<T_{d}^{0}$ and $J-0.75$. This quench injects a smaller amount of energy into the system $\Delta e-0.208$. The post-quench critical temperatures are $T_{d}=0.159$ and $T_{s}-0.439$. Differently from the previous case, the correlations with the initial time $t_{2}-0^{+}$and with a waiting time $t_{2}>0$ decay to non-vanishing values, $q_{0}$ and $q$, respectively. The asymptotic analysis condensed in the full set of equations (85)-(87) predicts $q=0.500$ and $q_{0}=0.548$ that are in very good agreement with the values obtained with the numerical solution of the dynamic equations shown in figure 6(a).

In panel figure 6(b) we display the $\chi(C)$ parametric plot for a long waiting time $t_{2}=11.25$, that finds good agreement with the FDT at the final temperature $T_{f}=0.514$ predicted by the asymptotic analysis. As a complement we also plot the parametric construction for a very short waiting time, $t_{2}-0.0025$, to demonstrate that, for $C\left(t_{1}, t_{2}\right)$ very close to one, the slope is determined by the initial temperature $T^{\prime}$ instead of $T_{f}$. It is only after a transient that the FDT with the final temperature $T_{f}$ establishes.

The results in the previous paragraph are consistent with the fact that the energies reach their asymptotic values only after a (short) transient. From the energetic dynamics we observe that $e_{\text {kin }}^{f}-T_{f} / 2$ after $t_{\text {tr }} \simeq 1$ and, $e_{\text {pot, }}^{f}$, measured after the same transient, is also in very good agreement with the predictions of the asymptotic analysis, once the non-vanishing values of $q$ and $q_{0}$ are taken into account.

The temperature at which the TAP state in which the system was initialised, modified by the quench, disappears is $T_{\mathrm{TAP}}^{\max }-0.559$, that is slightly above the final temperature $\%_{f}=0.511$. Consequently, the analysis in section 4.1 .5 applies to this case and, after the quench, the system follows the TAP state in which it was set in initially.

5.2.3. $T_{s}^{0}<T^{\prime}<T_{d}^{0}$ : from $T A P$ to spinodal, transient dynamics. So far we have been interested in describing the asymptotic state of the system after the quench. We have shown that these asymptotic states can be described in terms of algebraic equations involving a few variables. Such asymptotic equations were derived inserting appropriate Ansatze in the full evolution equations. However, a systematic investigation of the full dynamical equations shows that there are parameter regimes in which 
Non equilibrium dynamics of isolated disordered systems: the classical Hamiltonian p-spin model
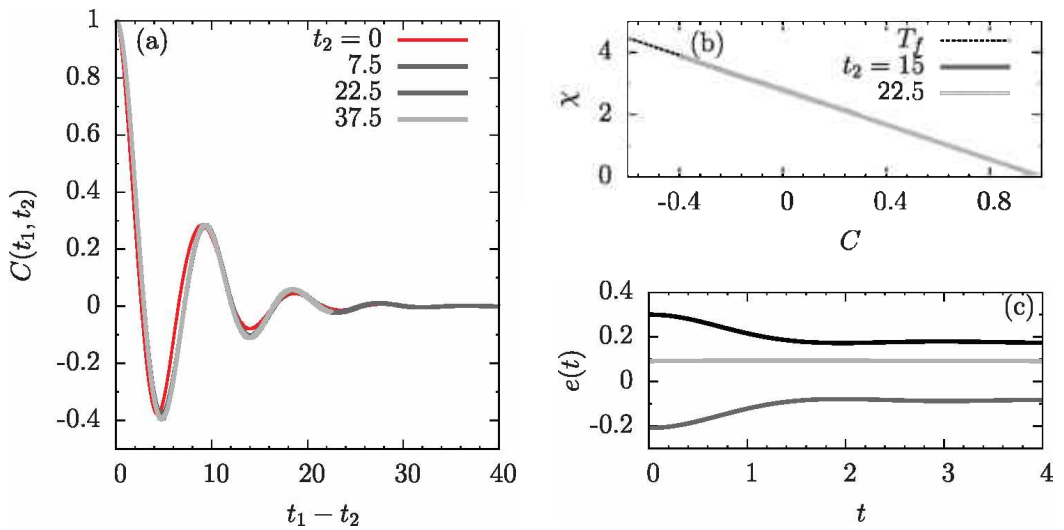

Figure 5. Numerical evolution for a quench from an initial state with temperature in the non-trivial interval, $T_{s}^{0}<T^{\prime}=0.6<T_{d}^{0}$ and $J_{0}=1, m_{0}$. The final parameters are $J-0.25$ and $m-1$. The energy injection is large, $\Delta e-0.625$. (a) Dynamics of the correlation function. The curves approach $q=0$ and $q_{0}=0$ as predicted by equations (85)-(87), indicating a paramagnetic equilibrium state. (b) The parametric plot. The black dashed line shows the FDT relation with $T_{f}-0.358$ as predicted by equation (91). The system reaches equilibrium at this new temperature. The solid lines correspond to the numerical results for two values of $t_{2}$. (c) Energy time-dependence. From top to bottom: kinetic energy (with stationary value $e_{\text {kin }}^{f}=0.179$ ), total energy (constant in time with value $\epsilon_{f}=0.092$ ) and potential energy (with stationary value $\epsilon_{\text {pot }}^{f}=-0.087$ ). Note that $c_{\text {kin }}^{f}=T_{f} / 2$ and $c_{\text {pot }}^{f}=-J^{2} /\left(2 T_{f}\right)$.

the system shows long lived transient dynamics before reaching the asymptotic state. This transient effects cannot be captured by the asymptotic analysis of the evolution equations.

We find transient dynamics near the interphases that separate the different asymptotic regimes. More precisely, near the interphase dividing the dynamics within TAP states from the dynamics that leaves the TAP state into the PM state, that is to say, close to the spinodal.

In figure 7 we show an example in which, starting from equilibrium below $T_{d}^{0}$, we inject encrgy and we observe a very long transient regime in which the system has non-stationary dynamics, with the correlations decaying faster for longer waiting times but not yet reaching the steady state, see figure 7(a). The non-stationary relaxation is accompanied by a waiting-time dependence of the parametric $\chi(C)$ plot. For short waiting times the parametric plot shows a piecewise form characteristic of ageing systems figure $7(\mathrm{~b})$. However, in this case, $T_{f}\left(l_{2}\right)>T_{\text {eff }}\left(l_{2}\right)$. In fact, a linear fit (shown with dotted lines in the figure) yields $T_{f}\left(t_{2}\right)=0.423$ and $T_{\text {eff }}\left(t_{2}\right)=0.349$. This behaviour persists for a finite period of time before slowly approaching the asymptotic $T_{f}$ in the whole range of variation of $C$ (not shown).

The asymptotic state should be paramagnetic for these parameters. Therefore, the expected $T_{f}$ is given by equation (91), and takes the value $T_{f}-0.41$. The predicted potential energy from the asymptotic analysis is $e_{\text {pot }}^{f}=-0.355$, that is in very good 
Non equilibrium dynamics of isolated disordered systems: the classical Hamiltonian p-spin model
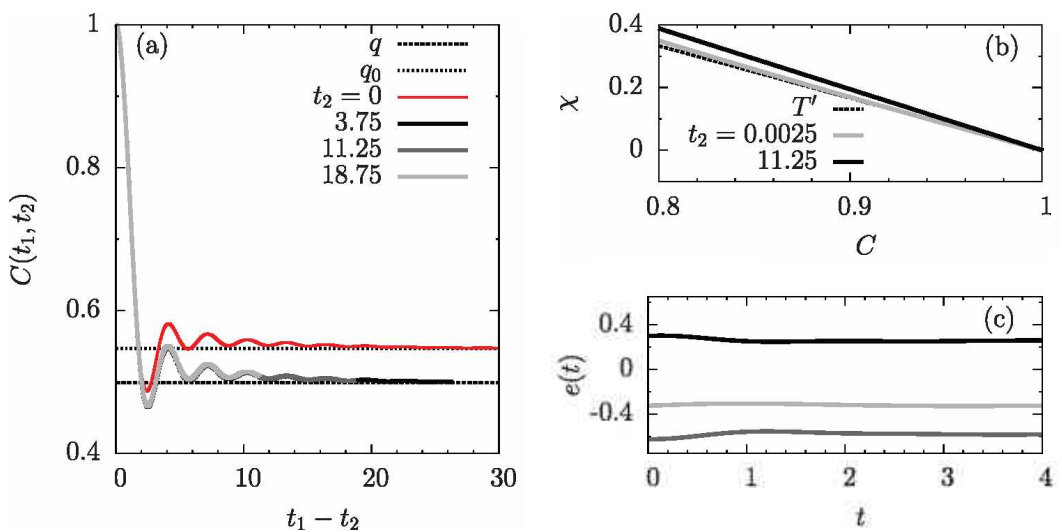

Figure 6. Initial temperature in the non-trivial interval, $T_{s}^{0}<T^{\prime}-0.6<T_{d}^{0}$. $m-m_{0}$ and $J-0.75$. The energy injection is small, $\Delta e-0.208$, and the system remains trapped in a TAP state. (a) Dynamics of the correlation function. The horizontal lines correspond to the asymptotic valnes $q=0.500$ and $q_{0}=0.548$, predicted by equations (85)-(87). (b) The parametric plot. The black dotted line shows the FDT relation with $T^{\prime}=0.6$ and it is compared to the numerical results at a very early time after the quench, $t_{2} \simeq 0.0025$. The other numerical line was obtained with $t_{2}=11.25$ a sufficiently long waiting time such that the asymptotic $T_{f}=0.514$ predicted by equations (85)-(87) is right below the data. (c) Energy timedependence. From top to bottom: kinetic energy (with stationary value $e_{\text {kin }}^{f}-0.257$ and in good agrement with $\epsilon_{\mathrm{kin}}^{f}=T_{f} / 2$ ), total encrgy (constant in time with value $e_{f}-0.092$ ) and potential energy (with stationary value $e_{\text {pot }}^{f}-0.582$ in agreement with equation (73) and differently from $-J^{2} /\left(2 T_{f}\right)$ ).

agreement with the numerical steady state value attained already at $\iota_{1} \simeq 2$ within numcrical accuracy. The kinetic energy also rcaches a platean after the same short transient (see the panel figure $7(\mathrm{c})$ ). We note that these two 'one-time' observables saturate much sooner that the correlation and linear response 'two-time' quantities converge to their final form.

The final temperature $T_{f}=0.41$ predicted by the asymptotic analysis is slightly above $T_{\mathrm{TAP}}^{\max }-0.402$, which justifies the PM nature of the asymptotic behaviour. However, the long-lived transient masks the PM behaviour at not sufficiently long times.

This behaviour shares points in common with observations already made in studies in different fields. In the context of quantum quenches, to have non-trivial dynamics of the correlation functions while quantities such as the kinetic energy has already thermalised is close to the concept of prethermalisation [42]. In the context of glassy physics, an asymptotic stationary decay in two steps, a faster one towards a platcau and a slower one towards zero is the kind of relaxation found in super-cooled liquids, the hallmark of the random first order phase transition scenario. Here we see that the correlation decays towards a value that is close to $q_{\mathrm{TAP}}^{\max }=(p-2) / p=1 / 3$, and it oscillates a few times around this value to later decay to zero, signalling the discontinuous way in which the TAP states disappear. Finally, the inversion in the temperature hierarchy, $T_{\text {eff }}\left(t_{2}\right)<T_{f}\left(t_{2}\right)$, found at short waiting times $t_{2}$ is a transient feature that shows the memory of the initial state with lower potential energy. In the dissipative problem, 
Non equilibrium dynamics of isolated disordered systems: the classical Hamiltonian p-spin model
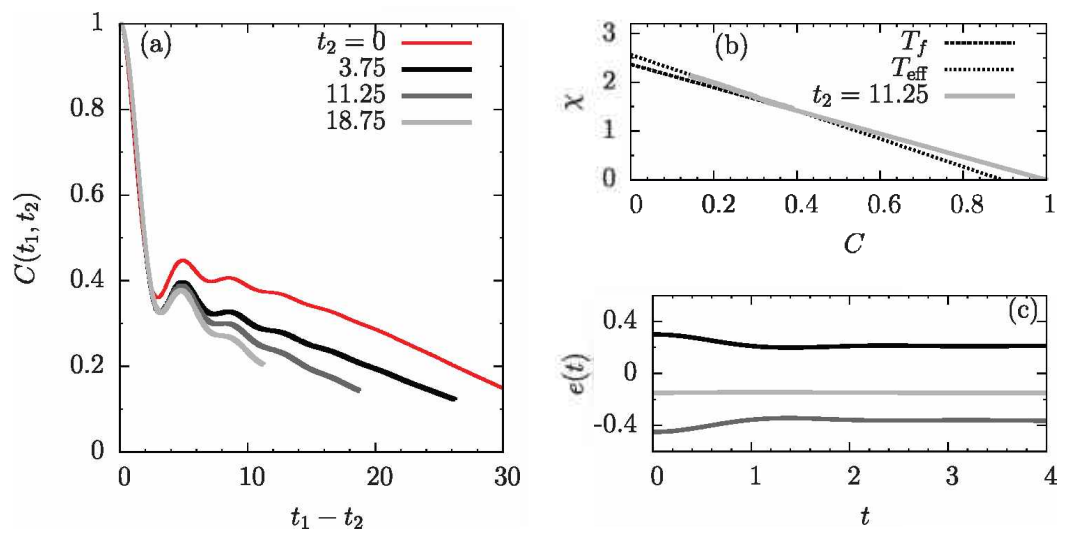

Figure 7. Initial temperature in the non-trivial interval, $T_{s}^{0}<T^{\prime}=0.6<T_{d}^{0}$, $m-m_{0}$ and $J-0.54$. The energy injection is $\Delta \epsilon-0.380$. (a) Dynamics of the correlation function. TTI is broken for these waiting times $t_{2}$. As the reference time increases, the correlation function relaxes more quickly, indicating that this behaviour is a transient. (b) Parametric plot. With solid gray line as the numerical results for $t_{2}-11.25$. With black dashed lines as the fits to the numerical data at short and long time differences yielding $T_{f} \neq T_{\text {cff }}$. (c) Energy time-dependence. From top to bottom: kinetic energy (with stationary value $e_{\text {kin }}^{f}=0.206$ ), total energy (constant in time with value $e_{f}=-0.15$ ) and potential energy (with stationary value $\epsilon_{\text {pot }}^{f}-0.356$ ).

$T_{\text {eff }}>T$ for quenches from the disordered to the low temperature phase $\left(T<T_{d}\right)$ and $T_{\text {eff }}<T$ for the dynamics in the low temperature phase for systems initiated in equilibrium at still lower temperatures than the one at which the dynamics takes place. This hierarchy is interpreted arguing that the effective temperature keeps memory of the initial state being more disordered or more ordered than the target one. This behaviour has been found in numerical simulations of the out of equilibrium dynamics of the $2 d$ xy model [43], an elastic line in a random potential [44] and atomic glass models [45], for instance. In the quenches we consider in this paper we only see the inversion in a pre-asymptotic regime.

5.2.4. $T_{s}^{\mathrm{U}}<T^{\prime}<T_{d}^{0}$ : from TAP to threshold? A natural question to pose is whether it is possible to take the system out of a TAP state and put it on the threshold level by injecting an adequate amount of energy. The asymptotic equations derived in section 4.2 under the assumption that this is possible allow for a non-trivial solution in a selected range of parameters. However, for the same set of parameters the stationary state equations that describe the dynamics within TAP states also admit non-trivial solutions. See figure 2 (b) where the TAP branch co-exists with the double ageing one. The complete numerical solution of the exact dynamic equations should then decide which of the two asymptotic states is actually rcalised. We have checked this issuc for, for example, $T^{\prime}=0.6, J_{0}-1, J=0.85$ and $m-m_{0}-1$, parameters such that the ageing asymptotic solution has $q \neq 0, q_{0}-0, T_{f}-0.542$ and $T_{\text {eff }}^{\prime}=0.148$ while the stationary state solutions are $q=0.55, q_{0}=0.574$ and $T_{f}=0.548>T_{d}$. The numerical analysis 
Non equilibrium dynamics of isolated disordered systems: the classical Hamiltonian p-spin model

of the full equations converges to the second option, showing that it is not possible to take the system out of a TAP state and put it on the threshold.

\subsection{Energy extraction}

When extracting energy with the quench $\left(I>J_{0}\right.$ and $\left.m-m_{0}\right)$, we will distinguish the cases in which the initial temperature is below or above the dynamic critical temperature $T_{d}^{0}$. We recall that in the former case the initial configuration is drawn from a non-trivial TAP state while in the latter it is simply paramagnetic. By extracting a small amount of energy from a highly energetic PM state the system remains in the PM state; these cases are not particularly interesting and we do not show any example of such. Instead, we focus on more interesting cases that are summarised in figure 11.

5.3.1. $T_{s}^{0}<T^{\prime}<T_{d}^{0}$ : from $T A P$ to $T A P$. In figure 8 we show results for $T_{s}^{0}<T^{\prime}-0.6<T_{d}^{0}$ and $J-1.5$. This quench extracts a large amount of energy from the system $\Delta e=-0.416$. For such value of $J$ the critical temperatures are $T_{d}=0.918$ and $T_{s}-0.440$. The final temperature $T_{f}-0.766$ and the parameters $q-0.680$ and $q_{0}=0.639$ predicted by the asymptotic analysis are in very good agreement with the results from the numerical solution of the complete equations. In this case $T_{s}<T_{f}<T_{d}$. From the energetic evolution we observe that $e_{\text {kin }}^{f}=T_{f} / 2$. In parallel, the potential encrgy $e_{\text {pot }}^{f}$ is also in very good agreement with the predictions of the asymptotic analysis using the non-vanishing values of $q$ and $q_{0}$.

This case is an example in which the initial TAP state is followed by the dynamies. The description in section 4.1 .5 applies and explains the results.

5.3.2. $T^{\prime}>T_{d}^{0}$ : from $P M$ to threshold, ageing dynamics. We will now demonstrate that for quenches from the paramagnetic state, $T^{\prime}>T_{d}$, with sufficient extraction of energy the system approaches the threshold level, similarly to what has been been observed in the past for the relaxation dynamics of the model coupled to a thermal bath. Due to the flatness of this region of phase space we observe ageing phenomena with the appearance of an effective temperature, and the subsequent non-validity of the fluctuation dissipation theorem, even with conserved energy dynamics.

In figure 9 we show results for energy extraction starting from a paramagnetic state, $T^{\prime}>T_{d}^{0}$, and using $J=1$, a value for which $T_{d}=2.324$ and $T_{s}=2.449$.

It is clear from panel figure 9 (a) that the correlation function does not reach a stationary regime; hence, time-translational invariance is broken. Moreover, the system ages since the curves for longer waiting times decay in a slower manner than the ones for shorter waiting times. The correlation shows oscillations at small values of the timedelay and these progressively disappear at long values of the same time-delay. The decay of any of the curves for different waiting times, but especially the ones for long waiting time, occurs in two steps.

The parametric plot $\chi(C)$ in figure $9(b)$ does not show a waiting-time dependence, as proven by the fact that the curves for two values of $t_{2}$ fall on top of each other. The resulting master curve also has a two step structure, with two slopes, which are in very good agrecment with the results from the asymptotic equations equations (106), (107) and (109) for $T_{f}$ and $T_{\text {eff. }}$ The breaking point in the piece-wise straight line is at $C \simeq 0.6$, 
Non equilibrium dynamics of isolated disordered systems: the classical Hamiltonian p-spin model
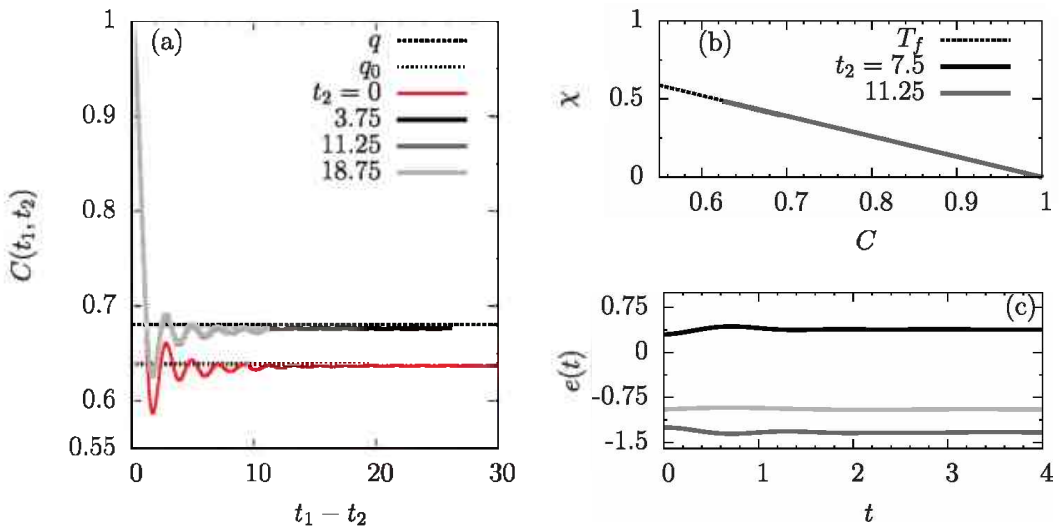

Figure 8. Starting from $T_{s}^{0}<T^{\prime}=0.6<T_{d}^{0}, m-m_{0}$ and we use $J-1.5$. The energy extraction is $\Delta e--0.416$. (a) Dynamics of the correlation function. The horizontal dotted lines correspond to the asymptotic values $q=0.680$ and $q_{0}=0.639$, predicted by equations (85)-(87). (b) Parametric $\chi(C)$ plot. The solid lines are the numerical results, in perfect agreement with the analytic prediction for the FDT with $T_{f}=0.766$, shown with a black dashed line. (c) Energy time-dependence. From top to bottom: kinetic energy (with stationary value $e_{\mathrm{kin}}^{f}=0.383=T_{f} / 2$ ), total energy (constant in time with value $e^{f}=-0.950$ ) and potential energy (with stationary value $\left.e_{\text {pot }}^{f}=-1.333\right)$.
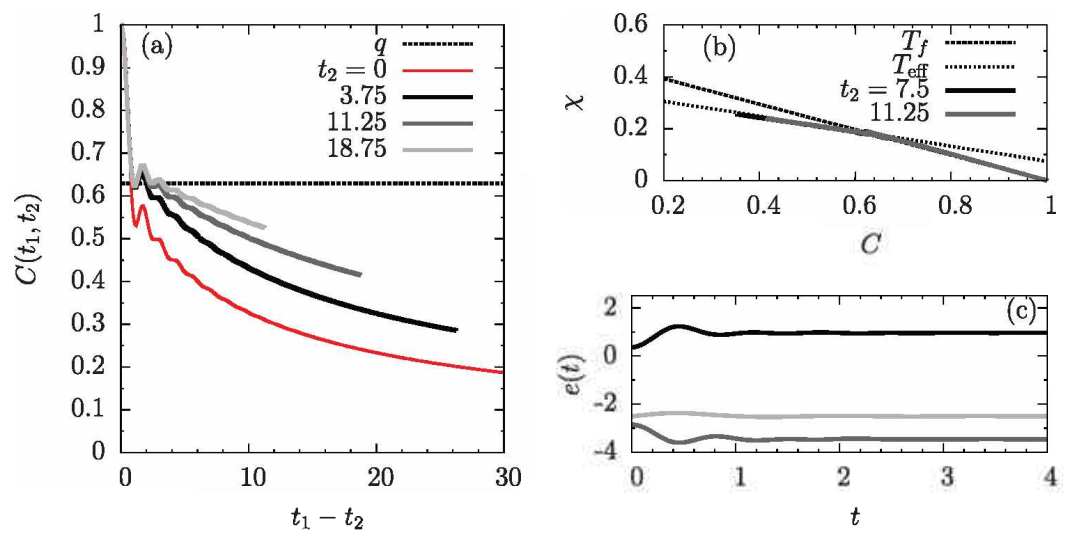

Figure 9. Starting from $T^{\prime}-0.7>T_{d}^{0}$ and $m_{0}$ we choose $m=m_{0}$ and $J-4$. The energy extraction is $\Delta e=-2.175$. (a) Dynamics of the correlation function. TTI is broken and ageing is evident. The dashed horizontal line corresponds to the value of $q$ predicted by the asymptotic equations, equations (106). (107) and (109). (b) Parametric plot. Black dashed and dotted lines are the predictions from the asymptotic equations with $T_{f}=2.036$ and $T_{\text {cff }}^{\prime}=3.461$. We also show numerical results for two different waiting times that are indistinguishable on the plot. (c) Energy time-dependence. From top to bottom: kinetic energy (with stationary value $\left.e_{\text {kin }}^{f}=0.995\right)$, total energy (constant in time with valne $e_{f}=-2.505$ ) and potential energy (with stationary value $\epsilon_{\mathrm{p} x \mathrm{t}}^{\prime}=-3.501$ ). 
Non equilibrium dynamics of isolated disordered systems: the classical Hamiltonian p-spin model $J<J_{0}$

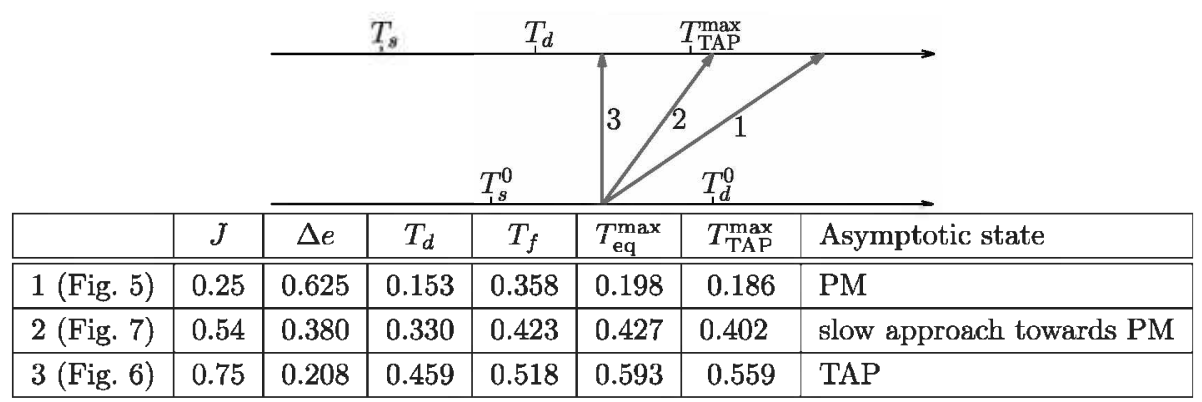

Figure 10. Schematic representation of the initial and final states of the quenches with energy injection studied numerically in figures 5 and 6 . The numbered labels refer to the entries in the table, in which we show quantitative information for each illustrative case.

which agrecs with the valuc of $q$ predicted by the asymptotic cquations, $q=0.629$, and the value of the change in behaviour of the two-time correlation, see panel figure 9(a).

Panel figure $9(\mathrm{c})$ shows the evolution of the two contributions to the energy density. We verify that the asymptotic kinetic energy density in figure $9(\mathrm{c})$ is consistent with $T_{f} / 2$. Moreover, the stationary potential energy density found numerically is also in very good agreement with the prediction from equation (108), $e_{\mathrm{pot}}^{f}=e_{\mathrm{th}}=-3.525$.

For intermediate energy extraction the system explores regions near the threshold level but still in the PM part of the landscape. As a consequence, there appear transient regimes in the dynamics at short times with non-stationary correlations that resemble the ageing ones, and a $\chi$ versus curve that can be characterised with two temperatures. However, for longer waiting times the dynamics converge to the asymptotic PM solution.

\subsection{Summary}

The results of the energy injection process are summarised in the table included in figure 10. The simplest way to understand what is going on is to compare the final temperature $T_{f}$ to the characteristic temperatures after the quench. In the three cases shown, $T_{f}>T_{d}$. However, the comparison between $T_{f}$ and $T_{\text {TAP }}^{\max }$ that corresponds to the spinodal line (see figure 1) explains the different behaviour in the three quenches. For $J-0.25, T_{f}>T_{\text {TAP }}^{\max }$ and the only possibility is to have a paramagnetic behaviour, as seen in figure 5 . For $J=0.54, T_{f} \gtrsim T_{\Gamma A P}^{\max }$ for the TAP state in which the system was initialised and, therefore, the system needs a long time to relax to the PM solution, see figure 7 . Finally, for $J=0.75$ the TAP state still exists after the quench and the system just follows it, as explained in section 4.1.5, see figure 6 .

We recap the two observations made for the energy extraction process in figure 11. The distinction is due to the initial state, being above or below $T_{d}$. In the latter case the system can only follow the TAP state in which it was prepared. In the former the parameters can be tuned to set it on the threshold. 


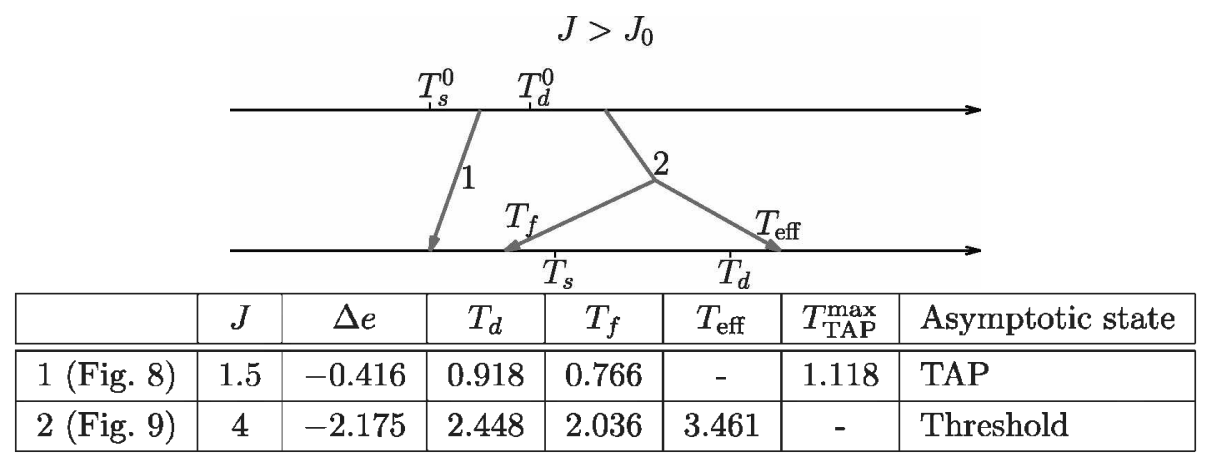

Figure 11. Schematic representation of the initial and final states of the quenches with energy extraction studied numerically in figures 8 and 9. The numbered labels make reference to the entries of the table, in which we show quantitative information for each illustrative case.

\section{The phase diagram}

The purpose of this section is to determine a dynamical phase diagram. We choose as the vertical axis the temperature at which the initial condition was equilibrated normalised by the parameter $J_{0}$. The horizontal axis is the control parameter of the quench that, as we will show, turns out to be $J m_{0} /\left(J_{0} m\right)$. We will show the regions in which the final state is either paramagnetic, a TAP state or a non equilibrium ageing state. As in the rest of this paper we show results for initial states equilibrated at temperatures $T_{s}^{0}<T^{\prime}$ only, so the origin of this axis is at $T_{s}^{0}$. In the rest of the section we explain the criteria used to obtain the critical lines in figure 12.

\subsection{The PM-ageing boundary line}

Initial states with $T^{\prime}>T_{d}^{0}$ are paramagnetic. We have seen in section 5.3 that extracting a small amount of energy, leaves the state in the paramagnetic region. Instead, by extracting a larger amount of energy it is possible to put the system on the threshold and, accordingly, the system displays ageing dynamics and is characterised by $T_{\text {eff }}>T_{f}$. If we claim that this second option ceases to be possible when $T_{\text {eff }}=T_{f}$, we can then use equation (107) to derive the value of $q$ on the transition line.

$$
1=(p-2)\left(1-q_{\mathrm{cr}}\right) / q_{\mathrm{cr}} \quad \Rightarrow \quad q_{\mathrm{cr}}=\frac{p-2}{p-1},
$$

that is $q_{\mathrm{th}}\left(T_{d}\right)$. If we now replace this $q_{\mathrm{cr}}$ in equation (106) and we use the constant $a(p)$, sce equation (25),

$$
T_{f_{\mathrm{cr}}}^{2}=J^{2} a^{2}(p)=T_{d}^{2},
$$

independently of $T^{\prime}$ and $J_{0}$, see figure 2. Still, what we are looking for is the curve $T^{\prime}\left(J_{0}, J\right)$ on which $T_{f}$ takes this value. We obtain it from equation (109) evaluated at $T_{f}=T_{\text {eff }}=T_{d}$ and $q=q_{\mathrm{cr}}$ 
Non equilibrium dynamics of isolated disordered systems: the classical Hamiltonian p-spin model
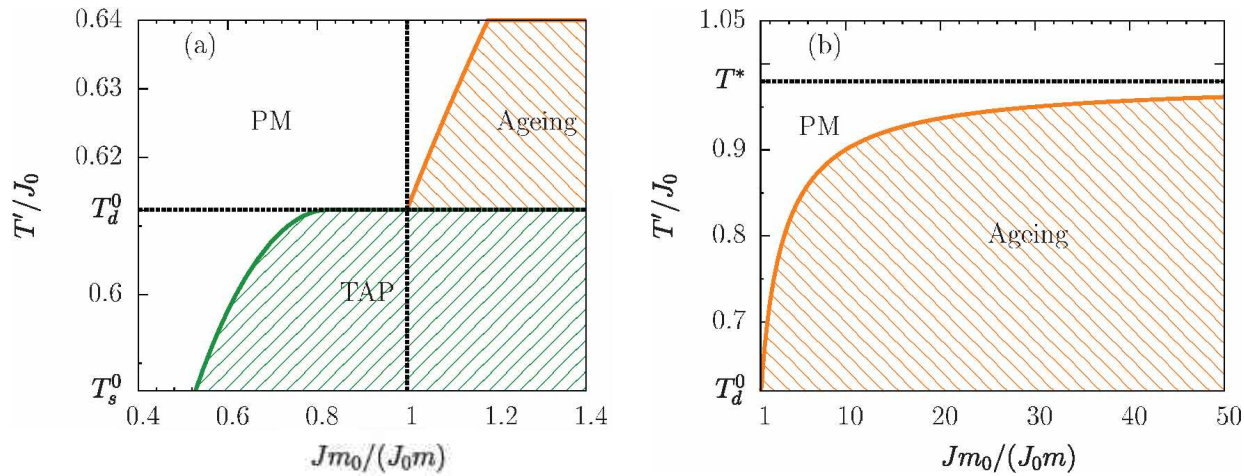

Figure 12. The phase diagram $\left(J m_{0} /\left(J_{0} m\right), T^{\prime} / J_{0}\right)$ with $T^{\prime}$ the temperature of the initial condition $J_{0}$ and $J$ the parameters that characterise the width of the disorder distribution before and after the quench, and $m_{0}$ and $m$ the mass of the particle before and after the quench. $J m_{0} /\left(J_{0} m\right)>1$ represents energy extraction and $J m_{0} /\left(J_{0} m\right)<1$ energy injection. In (a) we show the region around $T^{\prime}-T_{d}^{0}$ (low temperatures), while in (b) we show the region $T^{\prime}>T_{d}^{0}$. The analytic expressions for the boundary lines are obtained in this section.

$$
\frac{T^{\prime \prime}}{J_{0}}=\frac{1}{2} \frac{1}{a(p)} \frac{J m_{0}}{J_{0} m}\left[a^{2}(p)-1+\sqrt{\left(a^{2}(p)-1\right)^{2}+4 a^{2}(p) \frac{J_{0} m}{J m_{0}}}\right] .
$$

A Taylor expansion around $J m_{0} /\left(J_{0} m\right)=1$ yields, to first order,

$$
\frac{T^{\prime}}{J_{0}}-a(p)+\left(\frac{J m_{0}}{J_{0} m}-1\right) \frac{a(p)}{1+a^{2}(p)}
$$

and, in the particular case $J m_{0} /\left(. J_{0} m\right)=1$,

$$
T^{\prime}=a(p) J_{0}=T_{d}^{0} .
$$

In the limit $J m_{0} /\left(m J_{0}\right) \rightarrow \infty$, using $a^{2}(p)<1$, equation (116) implies

$$
\frac{T^{\prime}}{J_{0}} \rightarrow \frac{T^{*}}{J_{0}} \equiv \frac{a(p)}{1-a^{2}(p)},
$$

a finite value for all $p$; in particular, $T^{\prime} / J_{0} \rightarrow 2 \sqrt{6} / 5$ for $p=3$. This seemingly unexpected result can be rationalised as follows. For increasing $T^{\prime}$, the initial kinetic energy (at $l_{1}=0^{\prime}$ ) grows as $T^{\prime \prime}$, while the initial potential energy vanishes as $-J_{0} J /\left(2 T^{\prime \prime}\right)$ and the energy extraction, in the case in which, for concreteness, we apply a quench in the potential, as $J_{0}\left(J_{0}-J\right) /\left(2 T^{\prime}\right)$. In the final ageing state the two temperatures $T_{f}$ and $T_{\text {eff }}$ are finite for finite $J$, as one can simply verify from the asymptotic equations. Accordingly, for finite $J$ there is a maximal value of $T^{\prime}$ beyond which the initial kinetic energy cannot be extracted by the chosen $J$ to put the system on the threshold. The paramagnetic solution does not have this problem since its $T_{f}$ is not bounded in the same way for quenches with $T^{\prime \prime} / J_{0}>T^{*} / J_{0}$. The system then remains in the PM state. 
Non equilibrium dynamics of isolated disordered systems: the classical Hamiltonian p-spin model

Contrary to this limitation, in the thermal quenches of the over-damped dissipative model, the system can get rid of its extra energy by releasing it to the environment and quenches from arbitrary high temperature initial conditions can approach the threshold and show ageing. Another interesting feature is that the final $T_{f}$ cannot take arbitrary low values.

\subsection{The TAP-PM boundary line}

Suppose now that we start in a TAP initial state. As we have seen, if we inject a small amount of energy, the system finishes in the same TAP state of the post-quench potential. Nevertheless, if we inject a larger amount of energy, the system can end up in a paramagnetic final state. The TAP-PM boundary line should be determined by the impossibility to follow the TAP initial state at the target $J$. Therefore, the transition occurs on the spinodal, where the TAP states simply cease to exist (see the blue line and open squares in figure 2(b)).

The critical line $\left(J m_{0} /\left(J_{0} m\right), T^{\prime \prime} / J_{0}\right)$ can then be derived by exploiting the results in section 4.1.5. First, equation (99) relates the initial $q\left[J_{0}, T^{\prime}\right]$ to the final one $q\left[J, T_{f}\right]$. We can use the, by now usual, analysis of the bell-shaped rhs to deduce that a solution with $q\left[J, T_{f}\right] \neq 0$ exists as long as $q \leqslant q_{\text {max }}=(p-2) / p$. Evaluating then the rhs at this value

$$
1-q\left[J_{0}, T^{\prime}\right]=\frac{2 J^{2}}{p T_{f}^{2}}\left(\frac{p-2}{p}\right)^{p-2} .
$$

with $q\left|J_{0}, T^{\prime}\right|$ given by equation (94) that we recall here written in a more convenient way

$$
\frac{p}{2}\left(q\left[J_{0}, T^{\prime}\right]\right)^{p-2}\left(1-q\left[J_{0}, T^{\prime}\right]\right)=\frac{T^{\prime 2}}{J_{0}^{2}} .
$$

We now need an equation to fix $T_{f}$. This should be derived from the asymptotic dynamics part, exploiting the fact that $q\left[J, T_{f}\right]-q_{\max }-(p-2) / p$. Take equation (85) as a starting point. The only unknown (apart from $T_{f}$ ) is $q_{0}^{p}$. We can use the energy balance equation (87) to extract $q_{0}^{p}$ and get from (85)

$$
\frac{J_{0} m}{J m_{0}} \frac{T^{\prime}}{J_{0}}-\frac{J_{0}}{T^{\prime}}=\frac{T_{f}}{J}-\frac{J}{T_{f}}-\frac{2}{p} \frac{q_{\max }}{1-q_{\max }} \frac{T_{f}}{J}+\frac{J}{T_{f}} \varphi_{\max }^{p-1} .
$$

This expression simplifies a little bit replacing $q_{\max }=(p-2) / p$ and $q_{\max } /$ $\left(1-q_{\max }\right)-(p-2) / 2$,

$$
\frac{J_{0} m}{J m_{0}} \frac{T^{\prime}}{J_{0}}-\frac{J_{0}}{T^{\prime}}=\frac{2}{p} \frac{T_{f}}{J}-\frac{J}{T_{f}}\left[1-\left(\frac{p-2}{p}\right)^{p-1}\right] .
$$

again a quadratic equation for $T_{f} / J$. Now we have to replace the solution for $T_{f} / J$ in equation (120), use this linear equation on $q\left[J_{0}, T^{\prime}\right]$ to get its dependence on the parameters and replace it in equation (121). This is an implicit equation that yields the curve $\left(J_{0} m /\left(J m_{0}\right), T^{\prime} / J_{0}\right)$ that marks the end of the TAP region of the phase diagram for $T_{s}<T^{\prime}<T_{d}^{0}$. 
We can see whether this boundary touches the value $T^{\prime}-T_{d}^{0}$ at the quench parameter $J m_{0} /\left(J_{0} m\right)=1$ or elsewhere by setting $T^{\prime \prime}=T_{d}^{0}=a(p) J_{0}$, with initial $q$ value $q\left[J_{0}, T^{\prime}\right]-(p-2) /(p-1)$. Equation $(120)$ can be used to determine $T_{f}$.

$$
\frac{T_{f}^{2}}{J^{2}}=2 \frac{p-1}{p}\left(\frac{p-2}{p}\right)^{p-2}=1\left(\frac{p-1}{p}\right)^{p} a^{2}(p)
$$

that replaced in equation (123) yields the critical value of $J m_{0} /\left(J_{0} m\right)$ :

$$
\left(\frac{J m_{0}}{J_{0} m}\right)_{\mathrm{cr}}=a^{2}(p)\left\{1+\frac{4}{p}\left(\frac{p-1}{p}\right)^{p / 2} a^{2}(p)-\frac{1}{2}\left(\frac{p}{p-1}\right)^{p / 2}\left[1-\left(\frac{p-2}{p}\right)^{p-1}\right]\right\}^{-1} .
$$

In the case $p=3$ one has $a^{2}(3)-3 / 8$ and

$$
\left(\frac{J m_{0}}{J_{0} m}\right)_{\mathrm{cr}} \approx 0.82
$$

that is smaller than one, as shown in figure 12 . This value of $\left(\frac{J m_{0}}{I_{0} m}\right)_{\text {cr }}$ provides the minimum energy injection needed to reach temperatures above the limit of existence of the metastable states close to the threshold level, see figure 1.

\section{Conclusions}

We studied the dynamical evolution of a classical disordered model subject to a quench. By endowing the system with an intrinsic dynamics, we were able to investigate the evolution of the isolated model, thus analysing issues of thermalisation and equilibration in a classical setting.

We showed that, depending on the parameters used in the instantaneous quench, an interacting classical disordered model can either reach equilibrium or remain out of equilibrium in two different manners: it can be confined in a metastable state evolving with stationary dynamics characterised by a single temperature related to the final energy, or it can evolve in the so-called threshold level with non-stationary dynamics characterised by two temperatures, similarly to what happens in the dissipative model. Figure 12 summarises the different phases and transition lincs in the phase diagram parametrized by the most important parameters of the initial condition $\left(T^{\prime} / J_{0}\right)$ and the adimensional control parameter of the quench, $J m_{0} /\left(J_{0} m\right)$. We therefore showed that dynamic phase transitions in close interacting systems are also realised classically.

In the context of quantum quenches, an out of equilibrium dynamic transition was found in the Hubbard model [46-49], the Bose-Hubbard model [50] and the $O(N)$ mode] [51-54]. It is characterised by the fact that long time averages display a singular behaviour and the order parameter vanishes when post-quench coupling parameter, say $U_{f}$, approaches a critical value $U_{f}^{d}$ with critical dynamics [53]. On one and another side of the critical parameter the asymptotic values of these time-averages take qualitatively different behaviours, as in a conventional phase transition. The dynamic phase transition can be a feature of the pre-thermalisation regime, as in [52], or a fully asymptotic property as in the $O(N)$ model in the large $N$ limit [54]. 
Non equilibrium dynamics of isolated disordered systems: the classical Hamiltonian p-spin model

Compared to these problems, in this paper we showed that dynamic phase transitions in quenched close interacting systems are also realised classically. The dynamical phase diagram in figure 12 summarises the different phases and transition lines.

Let us now explain, in some detail, why we claim that the non-integrable model that we analysed shows equilibrium and out of equilibrium asymptotic dynamics. In the case of a closed system, the process of thermalisation consists in the loss of memory of the details of the initial condition, and the approach to a state that can be completely characterised by the values of the conserved quantities. Equilibrium statistical mechanics indicates that such a state should be described by the microcanonical, canonical or grand-canonical ensembles in the thermodynamic limit, depending on the degree of isolation from the environment. Then, at least for generic non-integrable models, we can say that a closed system has thermalised if

- the dynamics has reached a stationary state,

- this state is described by one of the ensembles of statistical mechanics.

Assuming thermalisation, the temperature of the canonical distribution describing the asymptotic state of a system with constant particle number is univocally defined by its encrgy. More specifically, if $H$ is the Hamiltonian of the system and $\rho_{0}$ is the initial macrostate, the energy of the system is given by $E-\langle H\rangle_{0}$, where the subscript indicates that we are taking the statistical average with respect to $\rho_{0}$. The final canonical state is then $\rho_{\text {th }}=\mathrm{e}^{-\beta H} / Z$, with $\beta$ determined by $E=\langle H\rangle_{\text {th }}$, where the subscript indicates the thermal average.

In the model that we studied, we found that, depending on the quench parameters, the asymptotic dynamics occur on the threshold, within a TAP metastable state or in the paramagnetic state. We now explain why the first two cases are out of equilibrium while the latter is in equilibrium, according to the two conditions listed above.

In the parameter regimes in which the ageing solution is realised, it is quite clear that the system does not thermalise, simply because it never reaches a stationary state.

We now argue that whenever the dynamics of the system is confined in a TAP state, i.e. $q, q_{0} \neq 0$, although the asymptotic state is stationary and even satisfies FDT with respect to its asymptotic temperature $T_{f}$, the system does not thermalise in the sense discussed above. Let us consider two different initial conditions specified by the values of $J_{0}$ and $T^{\prime \prime},(1)=\left\{J_{0}^{(1)}, T^{\prime(1)}=0.6 J_{0}^{(1)}\right\}$ and $(2)=\left\{J_{0}^{(2)}=1.05 J_{0}^{(1)}, T^{\prime(1)}=0.62 J_{0}^{(1)}\right\}$, where we use $f_{0}^{(1)}$ as the energy unit. In both cases $T_{s}^{0}<T^{\prime}<T_{d}^{0}$, which means that the system is prepared in a TAP state. We will take the evolution Hamiltonian $H$ to be specified by $I-0.75 J_{0}^{(1)}$ in both cases. Now, using equation (61) it is easy to verify that both systems have the same energy density, $e_{f}-0.325 . J_{0}^{(1)}$. If the only conserved quantity is energy, that is the Hamiltonian $H$ itself, and both systems thermalise, the asymptotic states reached should be identical. However, the asymptotic states predicted by equations (85)-(87), that we have checked against the full dynamics, are different $\left\{q^{(1)}=0.5, q_{0}^{(1)}=0.548, T_{f}^{(1)}=0.513 J_{0}^{(1)}\right\}$ and $\left\{q^{(1)}=0.529, q_{0}^{(1)}=0.578, T_{f}^{(1)}=0.519 J_{0}^{(1)}\right\}$. In particular, the final temperatures are different. We can interpret this fact using the results in section 4.1.5. Both systems are initialised in a TAP state and, since we inject only a small amount of energy, the 
subsequent dynamics takes place inside the same TAP state that is only translated in energy and rescaled in size by the quench. In other words, both systems are unable to forget the details of the initial condition, in particular which is the initial TAP state. Both systems reach thermal equilibrium inside the TAP state, however this bounded equilibrium is not compatible with thermal equilibrium in the whole phase space. In fact, for both quenches the final temperature $T_{f}^{(1,2)}$ is larger than the dynamical temperature $T_{d}=0.459 J_{0}^{(1)}$ and both systems show $q \neq 0$. However, the equilibrium state described by the Gibbs measure $\mathrm{e}^{-\beta_{f} H} / Z$ is paramagnetic for any $T_{f}^{\prime}>T_{d}$, with $q=0$. This confirms that the asymptotic states reached by both systems are not thermal.

Finally, quenches ending up in a paramagnetic asymptotic state with $q=q_{0}=0$ show thermalisation. Let us consider two different initial conditions $(1)=\left\{J_{0}^{(1)}, T^{\prime(1)}=0.6 J_{0}^{(1)}\right\}$ and $(2)=\left\{J_{0}^{(2)}=1.05 . J_{0}^{(1)}, T^{(1)}=0.615 J_{0}^{(\mathbf{1})}\right\}$. Again, both initial conditions are prepared in a TAP state. Now we will consider a situation in which we inject a larger amount of energy by choosing $J-0.4 . J_{0}^{(1)}$. In this case, both systems have the same energy density $e_{f}--0.033 J_{0}^{(1)}$. The asymptotic state in both cases is paramagnetic and the final temperatures are also equal $T_{f}^{(1)}=T_{f}^{(2)}=0.368 J_{0}^{(1)}$. Since the final temperature is larger than the dynamical temperature $T_{d}=0.214 J_{0}^{(1)}$, the asymptotic state is compatible with the paramagnetic equilibrium state $\mathrm{e}^{-\beta_{f} H} / Z$.

We end with a discussion of the temperatures selected by the asymptotic state, be it a steady or an ageing one. The steady state can be a simple paramagnet or a metastable TAP state. In both of these cases, the model fixes its temperature, that is a function of the energy after the quench, and the fluctuation dissipation theorem is satisfied with respect to it. In the ageing asymptotic state, instead, the dynamics occur in two, well separated, time regimes controlled by the relation between the time-delay and the reference or waiting time. The fluctuation-dissipation relation is satisfied with respect to these two temperatures for the time-delay taking values in the two regimes. The crossover between the two regimes takes place when the correlation function passes by the plateau value $q$. The ageing regime has exactly the same features as for the dissipative system.

It was recently shown that in quenches of isolated quantum integrable systems the fluctuation dissipation relation, as a function of frequency, allows one to measure, in the steady state, the generalised Gibbs ensemble effective temperatures, one for each conserved quantity $[55,56]$. In the model we studied in this paper there is only one conserved quantity, the total energy, but, in the ageing asymptotic state, the system acquires two temperatures, depending on the range of frequencies at which it is examined. Whether there is a link between the temperatures ( $T_{f}$ and $T_{\text {eff }}$ ) measured in the out of equilibrium non-integrable systems and the ones of the GGE of integrable models, is a question that deserves further analysis.

The TAP states are separated by barriers diverging with the system size, that is to say, with $N$ in this case. In the dissipative problem the escape time from the TAP states is expected to grow exponentially, à la Arrhenius, with the surrounding (free-energy) barrier over the thermal energy $k_{\mathrm{B}} T$ and, therefore, exponentially with $N$. In the infinite size limit, the TAP states are fully confining even under thermal fluctuations. Numerical evidence for the finite (though very long) lifetime of the trapping states for finite $N$ was given in, e.g. $\lceil 38-40\rceil$. 
Non equilibrium dynamics of isolated disordered systems: the classical Hamiltonian p-spin model

As shown by our results, in the isolated problem, the asymptotic quench dynamics of the infinite size system can also be confined to these states with special choices of the initial conditions (that are correlated with the random potential). The calculation that we presented in this paper are valid only in the infinite size limit. In the isolated case, though, we expect that the remnants of the TAP states in the finite $N$ systems should be confining until some crossover energy input is exceeded; how this is achieved should depend on the parameters.

Under these conditions, a system initially prepared in a configuration belonging to a finite $N$ psendo-TAP state will initially explore the region of the phase space corresponding to the transformed finite $N$ pseudo-TAP state, before relaxing to the final paramagnetic state in a longer timescale. The crossover time should scale with $N$, becoming infinite for $N \rightarrow \infty$, in concordance with our results. This two step relaxation would be reminiscent of the situation in nearly integrable isolated quantum many body systems, which initially relax to a metastable state (called prethermalised state in the recent literature [57-59]) before reaching equilibrium in a longer time-scale [59]. Prethermalised states in nearly integrable systems are in correspondence with the nonthermal stationary states of the associated integrable model $[57,58]$. In this case, the crossover time-scale scales with the distance to integrability, and diverges for the integrable model, for which the non-thermal stationary state, often described by GGE density matrices, are the truly final stationary states. The picture of a two step relaxation should also apply to disordered finite-range non-mean-field models with metastable states separated by large but finite energy barriers. Finally, we would like to point out that the existence of non-thermal quasistationary states whose lifetime scales with the system size is a well known fact in the dynamics of non-disordered systems with long, but finite, range interactions [60].

In a separate publication we will discuss how the methods and results of this paper extend to the quantum problem.

\section{Acknowledgments}

We warmly thank D Abanin, G Biroli, T Grover, J Kurchan, A Tartaglia and M Tarzia for very useful discussions. LFC thanks the KITP Santa Barbara for hospitality during part of the preparation of this work. We acknowledge financial support from ECOSSud A14E01, PICS 506691 (CNRS-CONICET Argentina) and NSF under Grant No. PHY11-25915. LFC is a member of Institut Universitaire de France.

\section{References}

[1] Polkovnikov $\Lambda$, Sengupta K, Silva $\Lambda$ and Vengalattore M 2011 Rev. Mod. Phys. 83863

[2] Bloch I, Dalibard J and Zwerger W 2008 Rev. Mod. Phys. 80885

[3] Basko D M, Aleiner I L and Altshuler B L 2006 Ann. Phys. 3211126

[4] Calabrese P, Essler F H L and Mussardo G 2016 J. Stat. Mech. 064001

5) Nandkishore R and Huse D A 2015 Annu. Rev. Condens. Matter Phys. 615

[6] Altman E and Vosk R 2015 Annu. Rev. Condens. Matler Phys. 6383

[7] Huse D A 2016 Physics 976

|8| Kirkpatrick T R and Thirmmalai D 1987 Phys. Rev. Lett. 582091

[9] Kirkpatrick T R and Thirumalai D 1987 Phys. Rev. B 365388

[10] Kirkpatrick 'I' R and Wolynes P 1987 Phys. Rev. B 368552 
Non equilibrium dynamics of isolated disordered systems: the classical Hamiltonian p-spin model

[11] Derrida B 1980 Phys. Rev. Letl. 4579

[12] Gross D J and Mézard M 1984 Nucl. Phys. B 240431

[13] Baldwin C I, Jaumann C R, Pal A and Scardichio A 2016 Phys. Rev. B 93024202

[14] Baldwin C L, Laumann C R, Pal A and Scardicchio A 2016 Clustering of non-ergodic eigenstates in quantum spin glasses (arXiv 1611.02296$)$

[15] Kirkpatrick T R and Thirumalai D 2015 Rev. Mod. Phys. 87183

[16] Crisanti $\Lambda$ and Sommers H-J 1992 Z. Phys. B: Condons. Matter 87341

[17] Cugliandolo L F and Kurchan J 1993 Phys. Rev. Lett. 71173

[18] Cugliandolo L F and Lozano G S 1998 Phys. Rev. Lett. 804979

[19] Cugliandolo L F and Lozano G S 1999 Phys. Rev. B 59915

[20] Lingel A 1993 Nucl. Phys. B 410617

[21] Franz S and Mézard M 1994 Europhys. Lett. 26209

[22] Cugliandolo L F and Le Doussal P 1996 Phys. Rev. E 53152

[23] Barrat A 1997 The p-spin spherical spin glass model (cond-mat/9701031)

[24] Castellani T and Cavagna A 2005 J. Stat. Mech. P05012

[25] Crisanti A and Sommers H-J 1995 J. Phys. I 5805

[26] Rieger H 1992 Phys. Rev. B 4614655

[27] Kurchan J, Parisi G and Virasoro M $\Lambda 1993$ J. Phys. I 31819

[28] Cavagna A, Giardina I and Parisi G 1998 Phys. Rev. B 5711251

[29] Mehta D, Stariolo D A and Kastner M 2013 Phys. Rev. E 87052143

[30] Crisanti $\Lambda$, Horner H and Sommers H-J 1993 Z. Phys. B: Condens. Matter 92257

[31] Cugliandolo L F and Kurchan J 1995 Phil. Mag. B 71501

[32] Cugliandolo L F, Kurchan J and Peliti L 1997 Phys. Rev. E 553898

[33] Cugliandolo L F 2011 J. Phys. A: Math. Theor, 44483001

[31] Houghton A, Jain S and Young A P 1983 Phys. Rev. B 282630

[35] Thirumalai D and Kirkpatrick T R 1988 Phys. Rev. B 384881

[36] Franz S and Parisi G 1995 J. Phys. I 51401

[37] Barrat A, Burioni R and Mézard M 1996 J. Phys. A: Math. Gen. 29 L81

[38] Cugliandolo L F, Kurchan J, Doussal P L and Peliti L 1997 Phys. Rev. Lelt. 78350

[39] Berthier L, Cugliandolo L F and Iguain J L 2001 Phys. Rev. E 63051302

[40] Berthier L, 2003 J. Phys: Condens. Matter 15 S933

[41] Cugliandolo L F 2002 Les Houches Lecture Notes (Berlin: Springer)

[42] Berges J, Borsanyi S and Wetterich C 2004 Phys. Rev. Lett. 93142002

[43] Berthier T, Holdsworth P C W and Sellit to M 2001 .I. Phys. A: Math. Gen. 341805

[44] Iguain J L, Bustingorry S, Kolton $\Lambda$ B and Cugliandolo L F 2009 Phys. Rev. B 80094201

[45] Gnan N, Maggi C, Parisi G and Sciortino F 2013 Phys. Rev. Lett. 110035701

[46] Eckstein M, Kollar M and Werner P 2009 Phys. Rev. Lett. 103056403

[47] Schiró M and Fabrizio M 2010 Phys. Rov. Lott. 105076401

[48] 'T'suji N, Lskstein M and Werner P 2013 Phys. Rev. Lett. 110136404

[49] Tsuji N and Werner P 2013 Phys. Rev. B 88165115

[50] Sciolla B and Biroli G 2010 Phys. Rov. Lett. 105220401

[51] Gambassi A and Calabrese ए' 2011 Furophys. Lett. 9566007

[52] Sciolla B and Biroli G 2011 J. Stat. Mech. P11003

[53] Sciolla B and Biroli G 2013 Phys. Rev. B 88201110

[51] Maraga A, Chiocchetta A, Mitra A and Gambassi A 2015 Phys. Rev. F 92042151

[55] Foini L, Gambassi A, Konik R and Cugliandolo L F 2016 Measuring effective temperatures in a generalized gibbs ensemble (arXiv: 1610.00101)

[56] de Nardis J et al 2016 Probing non-thermal density fluctuations in the one-dimensional bose gas (arXiv: 1704.06649)

[57] Kollar M, Wolf F A and Lckstein M 2011 Phys. Rev. B 84054304

[58] Nessi N, Iucci A and Cazalilla M A 2014 Phys. Rev. Lett. 113210402

[59] Bertini B, Essler F H, Groha S and Robinson N J 2015 Phys. Rev. Lett. 115180601

[60] Gupta S and Ruffo S 2017 Int. J. Mod. Phys. A 321741018 